M. Furushima

Nagoya Math. J.

Tol. 104 (1986), 1-28

\title{
SINGULAR DEL PEZZO SURFACES AND ANALYTIC COMPACTIFICATIONS OF 3-DIMENSIONAL COMPLEX AFFINE SPACE $C^{3}$
}

\author{
MIKIO FURUSHIMA
}

\section{Introduction}

Let $X$ be an $n$-dimensional connected compact complex manifold and $A$ be an analytic subset of $X$. We say that the pair $(X, A)$ is a complex analytic compactification of $C^{n}$ if $X-A$ is biholomorphic to $C^{n}$. If $X$ admits a Kähler metric, we shall say that $(X, A)$ is a (non-singular) Kähler compactification of $C^{n}$. For $n=1$, it is easy to see that $(X, A) \simeq\left(\boldsymbol{P}^{1}, \infty\right)$. For $n=2$, Remmert-Van de Ven [17] proved that $(X, A) \simeq\left(\boldsymbol{P}^{2}, \boldsymbol{P}^{1}\right)$ if $A$ is irreducible, where $A=\boldsymbol{P}^{1}$ is linearly embedded in $\boldsymbol{P}^{2}$. Morrow [15] gave more detailed classifications of complex analytic compactifications of $C^{2}$. For $n=3$, Brenton-Morrow showed the following

TheORem ([5]). Let $(X, A)$ be a non-singular Kähler complex analytic compactification of $C^{3}$ such that the analytic subset $A$ has only isolated singular points. Then $X$ is projective algebraic and $A$ is birationally equivalent to a ruled surface over an algebraic curve of genus $g=b_{3}(X) / 2$.

Further, Brenton [3]* classified the possible types of singular points of $A$ in the case that the canonical line bundle $K_{.4}$ of $A$ is not trivial.

In this paper, we shall discuss in detail the structures of the nonsingular Kähler compactifications of $C^{3}$ such that $A$ has at most isolated singular points.

Our results can be summarized as follows

Theorem I. Let $(X, A)$ be a non-singular Kähler compactification of $C^{3}$ such that $A$ has at most isolated singular points. Then $A$ is an irreducible

Received December 10, 1983.

Revised August 27, 1985.

: We remark that there exist some ambiguities in the arguments in [3], but the results are justified later by Hidaka-Watanabe [9]. 
normal Gorenstein surface, the line bundle [A] defined by $A$ is positive on $X$, and $K_{X}=-r[A](1 \leqq r \leqq 4)$, where $K_{X}$ is the canonical line bundle of $X$. Further, the structure of $(X, A)$ is determined by $r$ in case $r \geqq 2$ as in the Table (I) below.

Table (I)

\begin{tabular}{|c|c|c|}
\hline$r$ & $(X, A)$ & \\
\hline 4 & $\left(\boldsymbol{P}^{3}, \boldsymbol{P}^{2}\right)$ & $A=\boldsymbol{P}^{2}$ is linearly embedded in $\boldsymbol{P}^{3}$. \\
\hline 3 & $\left(\boldsymbol{Q}^{3}, \boldsymbol{Q}_{0}^{2}\right)$ & $\begin{array}{l}\boldsymbol{Q}^{3} \text { is a non-singular quadric hypersurface in } \boldsymbol{P}^{+} \text {and } \\
\boldsymbol{Q}_{0}^{2} \text { is a quadric cone which is a hyperplane section. }\end{array}$ \\
\hline 2 & $\left(V_{5}, H_{5}\right)$ & $\begin{array}{l}V_{5} \text { is a } 3 \text {-fold of degree } 5 \text { in } \boldsymbol{P}^{6} \text {, and } H_{5} \text { is a hyper- } \\
\text { plane section with an isolated singular point. }\left(V_{5}, H_{5}\right) \\
\text { is uniquely determined up to isomorphism. The de- } \\
\text { tailed structure of }\left(V_{5}, H_{5}\right) \text { will described in Section } \\
4 \text { below. }\end{array}$ \\
\hline 1 & unknown & $\begin{array}{l}A \text { is not a cone over a compact algebraic curve with } \\
\text { genus } g \geqq 0 \text {. }\end{array}$ \\
\hline
\end{tabular}

The main part of our proof is sacrificed to the investigation of the structure of $A$. Particularly, we shall determine completely the structures of singular Del Pezzo surfaces $A$ with Pic $A \simeq Z$ (see $\S 3$ ).

After such an investigation of the boundary divisor $A$ and the normal bundle $N_{A}$ of $A$, the projecting method from a line contained in $A \longrightarrow X$ by Fano-Iskovski [11] enables us to prove the theorem (see $\S 4$ ). In the course of the proof of the case $r=2$ of our Theorem I, we find the following fact, which gives the negative answer to a question of BrentonMorrow [5, p. 151].

TheOREM II. There exists a non-singular projective algebraic compactifcation $(X, E)$ of $C^{3}$ such that $E$ is irreducible but not normal.

As for the case of $r=1$, we have some more detailed informations, but the complete structure of $(X, A)$ is not yet unknown for us for the present.

The author would like to express his hearty thanks to Prof. M. Suzuki and Prof. E. Sato for their invaluable suggestions and encouragement. 


\section{Contents}

$\S 1$. Preliminaries and the case of $r \geqq 3$.

(1.1) General properties.

(1.2) Minimal resolution of singular points of $A$.

(1.3) The case of $r \geqq 3$.

$\S 2$. Cone singularities and the compactification of $C^{3}$.

(2.1) The genus of the cone singularity.

(2.2) Structure of $(X, A)$.

(2.3) Remarks.

$\S 3 . \quad$ Sinuglar Del Pezzo surfaces with free Picard group of rank 1 .

(3.1) The two classes of singular Del Pezzo surfaces.

(3.2) Almost general position.

(3.3) Rational singular Del Pezzo surfaces with $\mathrm{Pic} \simeq Z$.

$\S 4$. The case of $r=2$.

(4.1) Non-existence of $(X, A)$ with $m=(A A A) \leqq 4$.

(4.2) The structure of $(X, A)$ with $m=5$.

(4.3) The construction of $(X, A)$ with $m=5$.

(4.4) Non-existence of $(X, A)$ with $m=6$.

(4.5) Conclusion.

\section{$\S 1$. Preliminaries and the case of $r \geqq 3$}

(1.1) General properties. Let $(X, A)$ be a non-singular Kähler compactification of $C^{3}$ such that $A$ has at most isolated singular points. At any point of $A$, one of the coordinate functions of $C^{3}$ must have a singularity. $A$ has therefore, by Hartogs theorem, pure dimension 2. Let us denote the set of regular points of $A$ by $A_{0}$. Since $A$ has at most isolated hypersurface singular points, the canonical line bundle $K_{A_{0}}$ on $A_{0}$ is trivial in a neighbourhood of each singular point of $A$. Such a singular point is called Gorenstein singularity. We call a complex surface with at most Gorenstein singularities a Gorenstein surface. Since $C^{3}$ is connected at infinity, $A$ is connected, and since $A$ is normal, $A$ is an irreducible normal Gorenstein surface. Let us consider the long exact sequence of cohomology groups corresponding to the pair $(X, A)$ :

$$
\longrightarrow H^{i}(X, A ; Z) \longrightarrow H^{i}(X ; Z) \longrightarrow H^{i}(A ; Z) \longrightarrow H^{i+1}(X, A ; Z) \longrightarrow
$$

Since

$$
H^{i}(X, A ; Z) \simeq H_{0-i}(X-A ; Z) \simeq H_{b-i}\left(C^{3} ; Z\right)=0 \quad \text { for } 1 \leqq i \leqq 5
$$


and $0 \rightarrow H^{5}(X ; Z) \rightarrow H^{5}(A ; Z) \simeq 0$, we have $H^{i}(X ; Z) \simeq H^{i}(A ; Z)$ for $i \leqq 5$.

Proposition 1 (Brenton-Morrow [p. 147, 5]).

(1) $H^{1}(X ; Z) \simeq H^{1}(A ; Z) \simeq 0$.

(2) $H^{2}(X ; Z) \simeq H^{2}(A ; Z) \simeq Z . \quad H^{2}(X ; Z)$ is generated by the first Chern class $c_{1}([A])$ of the line bundle $[A]$ defined by $A$ and $H^{2}(A ; Z)$ is generated by $c_{1}\left(N_{A}\right)$, where $N_{A}=\left.[A]\right|_{A}$ is the normal bundle on $A$.

(3) The Euler number $\chi(X)=4-b_{3}(A)$, where $b_{i}(A)=\operatorname{dim}_{R} H^{i}(A ; R)$. (because $H^{3}(X ; R) \cong H^{3}(A, R)$ by the argument before Proposition 1.)

(4) The line bundle $[A]$ is positive on $X$ and the canonical line bundle $K_{X}=-r[A](r>0)$. Especially $X$ is projective algebraic.

(5) $H^{i}\left(X ; \mathcal{O}_{X}\right)=0(1 \leqq i \leqq 3)$

Proposition 2. $H^{i}\left(A ; \mathcal{O}_{A}\right)=0$ for $1 \leqq i \leqq 2$ if $r \geqq 2$, and $H^{1}\left(A ; \mathcal{O}_{A}\right)=0$, $H^{2}\left(A ; \mathcal{O}_{A}\right) \simeq C$ if $r=1$.

Proof. Let us consider the following exact sequence:

$$
\begin{aligned}
\longrightarrow & H^{i}\left(X ; \mathcal{O}_{X}(-A)\right) \longrightarrow H^{i}\left(X ; \mathcal{O}_{X}\right) \\
& \longrightarrow H^{i}\left(A ; \mathcal{O}_{A}\right) \longrightarrow H^{i+1}\left(X ; \mathcal{O}_{X}(-A)\right) \longrightarrow
\end{aligned}
$$

By the Kodaira-Serre duality, $H^{i}\left(X ; \mathcal{O}_{X}(-A)\right) \simeq H^{3-i}\left(X ; \mathcal{O}_{X}\left(K_{X}+A\right)\right)$. Since $K_{X}+A=(1-r) A$, if $r \geqq 2$, then by Kodaira vanishing theorem, $H^{i}\left(X ; \mathcal{O}_{X}(-A)\right)=0$ for $i \leqq 3$. Thus $H^{i}\left(X ; \mathcal{O}_{X}\right) \simeq H^{i}\left(A ; \mathcal{O}_{A}\right)(1 \leqq i \leqq 2)$. Therefore, by Proposition 1 , we have $H^{i}\left(A ; \mathcal{O}_{A}\right)=0$. If $r=1$, then $K_{X}+$ $A \simeq \mathcal{O}_{X} . \quad$ Thus $H^{i}\left(X ; \mathcal{O}_{X}(-A)\right)=0 \quad(1 \leqq i \leqq 2)$. Therefore we have $H^{1}\left(A ; \mathcal{O}_{A}\right) \simeq H^{1}\left(X ; \mathcal{O}_{X}\right)=0$ and $H^{2}\left(A ; \mathcal{O}_{A}\right) \simeq H^{3}\left(X ; \mathcal{O}_{X}(-A)\right) \simeq H^{0}\left(X ; \mathcal{O}_{X}\right) \simeq C$.

Q.E.D.

Since $A$ is a normal Gorenstein surface, we can define the canonical line bundle $K_{A}$ on $A$ as the trivial extension of $K_{A_{0}}$ to $A$. By the adjunction formula, we have

$$
K_{A}=\left.K_{X}\right|_{A}+\left.[A]\right|_{A}=(1-r) N_{A}, \quad N_{A}=\left.[A]\right|_{A} .
$$

By Proposition 1-(4), $-K_{A}$ is positive on $A$ if $r \geqq 2$ and $K_{A} \simeq \mathcal{O}_{A}$ if $r=1$.

Definition 1. A normal Gorenstein surface $A$ will be called a (singular) Del Pezzo surface (resp. a singular $K-3$ surface) if $-K_{A}$ is positive on $A$ (resp. $-K_{A} \simeq \mathcal{O}_{A}$ and $H^{1}\left(A ; \mathcal{O}_{A}\right)=0$ ).

Thus we have the following 
Proposition 3. If $r \geqq 2$, then $A$ is a (singular) Del Pezzo surface with Pic $A \simeq Z\left(N_{A}\right)$. If $r=1$, then $A$ is a singular $K-3$ surface with Pic $A \simeq Z\left(N_{A}\right)$.

Proof. By Proposition 2 and the fact that $A$ is projective algebraic, we have $H^{1}\left(A ; \mathcal{O}_{A}^{*}\right) \simeq H^{2}(A ; Z) \simeq Z$.

Q.E.D.

(1.2) Minimal resolution of singular points of $A$. Let $x=\left\{x_{1}, \cdots, x_{r}\right\}$ be the set of singular points of $A$ and $\mu: M \rightarrow A$ be the minimal resolution of singularities of $A$. We put $\mu^{-1}(x)=\bigcup_{i=1}^{s} C_{i}$, where $C_{i}$ 's are the irreducible components of $C$. Since $A$ is projective algebraic, so is $M$. Let us denote the canonical line bundle of $M$ by $K_{Y}$. Since $\mu$ is the minimal resolution and $K_{A}=(1-r) N_{A}$, we have

$$
K_{M}=(1-r) \mu^{*}\left(N_{A}\right)-\sum_{i=1}^{s} n_{i} C_{i} \quad\left(n_{i} \geqq 0\right) .
$$

Proposition 4. Let $\bar{M}$ be a relatively minimal non-singular model of $M$, and $\nu: M \rightarrow \bar{M}$ be the birational morphism. Then $\bar{M}$ is either the projective plane $\boldsymbol{P}^{2}$ or the total space of a $\boldsymbol{P}^{1}$-bundle on a non-singular algebriac curve.

Proof (Brenton-Morrow [p. 148, 5]). We have only to show that $P_{m}(M)=$ $\operatorname{dim}_{C} H^{0}\left(M ; \mathcal{O}\left(m K_{x}\right)\right)=0$ for $m>0$, since $P_{m}$ is birational invariant among non-singular surfaces. But this follows from the inequality

$$
\begin{aligned}
\operatorname{dim}_{C} H^{0}\left(M ; \mathcal{O}\left(m K_{V}\right)\right) & \leqq \operatorname{dim}_{C} H^{0}\left(M ; \mathcal{O}\left((1-r) \mu^{*} N_{A}\right)\right) \\
& =\operatorname{dim}_{C} H^{0}\left(A ; \mathcal{O}_{A}\left((1-r) N_{A}\right)\right)
\end{aligned}
$$

and the fact that $b^{+}(M)=b^{-}(A)=1$ by Proposition 1-(2). Thus from the classification of surfaces [1], $\bar{M}$ is either $\boldsymbol{P}^{2}$ or the total space of a $\boldsymbol{P}^{1}$ bundle on a non-singular algebraic curve.

Q.E.D.

(1.3) The case of $r \geqq 3$.

Let us quote a theorem due to Kobayashi-Ochiai;

TheOREM (K-O). Let $Y$ be an n-dimensional compact complex manifold, and $L$ be a positive line bundle on $Y$. Then we have

(1) If $c_{1}(Y)=c\left(L^{k}\right)$ for some integer $k \geqq n+1$, then $Y$ is biholomorphic to $\boldsymbol{P}^{n}$.

(2) If $c_{1}(Y)=c\left(L^{n}\right)$, then $Y$ is biholomorphic to a non-singular quadric hypersurface $\boldsymbol{Q}^{n}$ in $\boldsymbol{P}^{n+1}$. 
By this theorem, we have

Proposition 5. (1) If $r \geqq 4$, then $A \cong M \cong \bar{M} \cong P^{2}$, consequently $r=4$. (2) If $r=3$, then $M=\bar{M}=\boldsymbol{F}_{2}$ and $\boldsymbol{A}=\boldsymbol{F}_{2} / \Gamma_{0} \cong \boldsymbol{Q}_{0}^{2} \longrightarrow \boldsymbol{P}^{3}$, where $\boldsymbol{F}_{2}$ is $a$ $\boldsymbol{P}^{1}$-bundle over a complex projective line $\boldsymbol{P}^{1}$ with the zero section $\Gamma_{0}$ with $\Gamma_{0}^{2}=-2$, and $\boldsymbol{Q}_{0}^{2} \longrightarrow \boldsymbol{P}^{3}$ is a quadric cone in $\boldsymbol{P}^{3}$.

Proof. (1): By Theorem (K-O), one has $X \cong P^{3}, r=4$, and $\mathcal{O}(A) \cong \mathcal{O}(1)$ because $r \geqq 4$. Thus $A \cong \boldsymbol{P}^{2} \cong M \cong \bar{M}$. (2): Similarly by Theorem (K-O), one sees $X$ is a quadric 3 -fold in $P^{4}$ and $\mathcal{O}(A) \cong \mathcal{O}_{X}(1)$ by $r=3$. Thus $A$ is a smooth quadric or $\boldsymbol{Q}_{2}^{0}$, and one sees $A \cong \boldsymbol{Q}_{0}^{2}$ because Pic $A \cong Z$.

Q.E.D.

One can easily see that $\boldsymbol{P}^{3}-\left\{\right.$ a hyperplane $\left.\boldsymbol{P}^{2}\right\} \cong C^{3}$ and $\boldsymbol{Q}^{3}-\{$ a hyperplane section $\left.\cong \boldsymbol{Q}_{0}^{2}\right\} \cong C^{3}$. Therefore we have the following

Proposition 6. (1) $r \geqq 4 \Leftrightarrow(X, A) \cong\left(\boldsymbol{P}^{3}, \boldsymbol{P}^{2}\right)$ (In fact, $\left.r=4\right)$,

(2) $r=3 \Longleftrightarrow(X, A) \cong\left(\boldsymbol{Q}^{3}, \boldsymbol{Q}_{0}^{2}\right)$.

\section{$\S 2$. Cone singularities and the compactifications of $C^{3}$}

(2.1) The genus of the cone singularity. Let $(X, A)$ be as in Section 1 . We say that $A$ has a cone singularity $\{x\}$ of genus $g$ if $\mu: M \rightarrow A$ being the minimal resolution, $M$ is a $P^{1}$-bundle over a non-singular algebraic curve $R$ of genus $g$ and $\mu^{-1}(x)=C$ is a section of this bundle.

Lemma 1. Let $\phi: M \rightarrow R$ be a $\boldsymbol{P}^{1}$-bundle over a non-singular algebraic curve $R$. Suppose that there exists a negative section $C_{0}$ and that the contraction $A=M / C_{0}$ of $C_{0}$ is algebraic. Then there exists a section $C_{\infty}$ such that $C_{\infty} \cap C_{0}=\phi$.

Proof. Since $A$ is algebraic, there exists another hyperplane section $D$ which does not pass through the singular point of $A$. In the surface $M$, we have $D \cap C_{0}=\phi$. For any point $z$ of $R$, we put $D \cap \phi^{-1}(z)=$ $\left\{p_{1}, \cdots, p_{\ell}\right\}$. Let $p_{z}$ be the barycentric point of the points $\left\{p_{1}, \cdots, p_{\ell}\right\}$ in $\phi^{-1}(z)-\phi^{-1}(z) \cap C_{0} \simeq C$. We put $C_{\infty}:=\bigcup_{z \in R} p_{z}$. Then we have a desired section $C_{\infty}$.

Q.E.D.

Proposition 7. If $A$ has a singularity of genus $g$, then the genus $g \leqq 3$ and the following holds

(i ) $r=3 \Longleftrightarrow g=0$.

(ii) $r=2 \Longleftrightarrow g=1$. 
(iii) $r=1 \Longleftrightarrow g=2$ or 3 .

Proof. Since $A$ is algebraic, by Lemma 1, there exists a section $D$ which does not intersect the negative section $C$, and we have $D^{2}=-C^{2}$. The curve $D$ can be considered as a Cartier divisor in $A$. Since Pic $A \cong$ $Z\left[N_{A}\right]$ by Proposition 3 and since $[D]$ is not divisible in Pic $A$ ( $D$ is pulled back to a section of $M$ by $\mu), N_{A}$ is linearly equivalent to the line bundle [D] defined by $D$. Since $K_{A}=(1-r) N_{A}$, by the adjunction formula, we have $K_{D}=\left.K_{A}\right|_{D}+N_{D}$, where $N_{D}=\left.[D]\right|_{D}$ is the normal bundle of $D$. By the Riemann-Roch theorem, we have the equation

$$
2 g-2=\operatorname{deg} K_{D}=(2-r) \operatorname{deg} N_{L} .
$$

Since $\operatorname{deg} N_{D}>0$ and $r \leqq 3$, we have (i) $r=3 \Leftrightarrow g=0$ and $\operatorname{deg} N_{L}=2$. (ii) $r=2 \Longleftrightarrow g=1$. In the case of $r=1$, one has $K_{A} \cong \mathcal{O}_{A}$ and $K_{I I}=$ $-2 C$ by the adjunction formula for a fibre. Let $L=N_{C}$ be the normal bundle of $C$ in $M$. Then $-L=-N_{C} \cong N_{D}$. By the adjunction formula, $K_{D}=\left.K_{u}\right|_{D}+N_{D}=N_{D}$. Since $(A, x)$ is a hypersurface singularity, emdim $(A, x)=3$. Let $m_{x}$ be the maximal ideal of the local ring $\mathcal{O}_{\perp, x}$. Since $\mathcal{O}_{A, x}$ is the localization of $\oplus_{k \geq 0} H^{0}(C ; \mathcal{O}(-k L))$ at the vertex, one

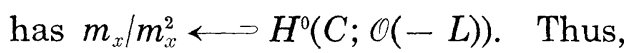

$$
\begin{aligned}
3 & =\operatorname{emdim}(A, x)=\operatorname{dim}_{C} m_{x} / m_{x}^{2} \geqq \operatorname{dim} H^{0}(C ; \mathcal{O}(-L)) \\
& =\operatorname{dim} H^{0}\left(D ; N_{D}\right)=\operatorname{dim} H^{0}\left(D ; K_{D}\right)=\mathrm{g},
\end{aligned}
$$

that is $g \leqq 3$. By the equation (*), we have (iii) $r=1 \Leftrightarrow g=2$ and $\operatorname{deg} N_{D}=2$, or $g=3$ and $\operatorname{deg} N_{D}=4$. This completes the proof. Q.E.D.

(2.2) Structure of $(X, A)$. In the case of $r=3$, we have determined the structure of $(X, A)$ in Section 1 . Therefore we have only to consider the case of $r \leqq 2$, to prove Theorem I.

In this case, $X$ is a Fano 3 -fold of index $r \leqq 2$ with $\operatorname{Pic} X \cong Z$.

We note that the topological Euler number of $A$ is written as

$$
\begin{aligned}
\chi(A) & =\chi(M)-\chi(C)+1 \\
& =2(2-2 g)-(2-2 g)+1=3-2 g,
\end{aligned}
$$

and $\chi(X)=\chi(A)+1=4-2 g$ because $\chi(X-A)=\chi\left(C^{3}\right)=1$. We will see that $\chi(X) \neq 4-2 g$ when $r \leqq 2$.

(Case 1) $\quad r=2$. By Proposition 7 , we have $g=1$, and thus $\chi(X)=2$. On the other hand, since $\chi(X)=4-b_{3}(X)=4-2 h^{1,2}$, by Table 3.5 in 
p. 809 in $\left[11_{\mathrm{a}}\right]$, we have $\chi(X)=-38,-16,-6,0,4$. This is a contradiction. Therefore $r \neq 2$.

(Case 2) $r=1$. We have then $g=2,3$. Thus $\chi(X)=0,-2$. Since $\operatorname{deg} N_{D}=2,4$ and since $\operatorname{deg} N_{D}=(A \cdot A \cdot A)_{X}=A^{3}$, by Table 3.5 in [11 $]$. $\chi(X)=-100,-56$. This is a contradiction. Therefore $r \neq 1$.

We note that we simply quoted a table in $\left[11_{a}\right]$ instead of going through lengthy calculation, to get $b_{3}(X)$ for Fano 3-folds $X$ with (1) index 2 , or (2) index 1 and $\left(-K_{X}\right)^{3}=2,4$. Because these are explicitly classified and $b_{3}(X)$ is well known among researchers in the field.

Thus we have the following

Theorem 1. Let $(X, A)$ be as in Theorem I. If $A$ is a cone over a compact algebraic curve of genus $g$, then $g=0(\Longleftrightarrow r=3)$ and $(X, A) \cong$ $\left(\boldsymbol{Q}^{3}, \boldsymbol{Q}_{0}^{2}\right)$.

\section{§3. Singular Del Pezzo surfaces with free Picard group of rank one}

(3.1) The two classes of singular Del Pezzo surfaces. Let $A$ be a singular Del Pezzo surface with isolated singularities $x=\left\{x_{1}, \cdots, x_{m}\right\}$ (see Definition 1 in $\S 1$ ). Let $\pi: M \rightarrow A$ be the minimal resolution of singularities of $A$ and put $\pi^{-1}(x)=C=\bigcup_{i=1}^{k} C_{i}$, where $C_{i}$ 's are irreducible components of $C$. Let us denote the canonical divisor on $M$ (resp. $A$ ) by $K_{M}$ (resp. $K_{A}$ ). Then we have

$$
K_{M}=\pi^{*} K_{A}-\sum_{i=1}^{k} n_{i} C_{i} \quad \text { (linearly equivalent), }
$$

where $n_{i} \geqq 0(1 \leqq i \leqq k)$.

Brenton and Hidaka-Watanabe showed the following

Proposition 9 ([3,9]). Let $A$ and $M$ be as above. Then we have either

(a) $A$ is the space obtained by blowing down the negative section $C_{0}$ of a $\boldsymbol{P}^{1}$-bundle $\bar{M}$ over a non-singular elliptic curve $T$, namely $A \cong \bar{M} / C_{0}$, or

(b) $A$ is a rational surface with only rational double points as singularities, obtained from $\boldsymbol{P}^{2}$ by blowing up $s$ (@8) points and then blowing down $s$ non-singular rational curves, each with the self-intersection number -2 .

(3.2) Almost general position. Let $\Sigma_{s}=\left\{p_{1}, \cdots, p_{s}\right\}$ be a finite set of points on the complex projective plane $\boldsymbol{P}^{2}$ (infinitely near points allowed) and assume that $s=\left|\Sigma_{s}\right| \leqq 8$. We put $\Sigma_{j}=\left\{p_{1}, \cdots, p_{j}\right\}(j \leqq s)$. Let $V\left(\Sigma_{j}\right)$ 
be the monoidal transformation with center $\Sigma_{j}$. Then we have the sequence

$$
M=V\left(\Sigma_{s}\right) \stackrel{\pi_{s}}{\longrightarrow} V\left(\Sigma_{s-1}\right) \longrightarrow \cdots \stackrel{\pi_{2}}{\longrightarrow} V\left(\Sigma_{1}\right) \stackrel{\pi_{1}}{\longrightarrow} \boldsymbol{P}^{2},
$$

where $p_{j+1} \in V\left(\Sigma_{j}\right)$. We put $E_{i}=\pi_{i}^{-1}\left(p_{i}\right)$.

Definition 2 (Demazure [6]). The points of $\Sigma_{s}$ are in almost general position if

(1) no four of them are on a line.

(2) no seven of them are on a conic.

(3) for all $j(1 \leqq j \leqq s-1)$, the point $p_{j+1} \in V\left(\Sigma_{j}\right)$ does not lie on any proper transform $\hat{E}_{i}$ such that $\hat{E}_{i}^{2}=-2$.

Then Demazure proved the following

Proposition 10 ([6]). (1) There exists a non-singular cubic curve $\Gamma_{0}$ which passes through all the points of $\Sigma_{s}$.

(2) The anti-canonical linear system $\left|-K_{M}\right|$ of $M$ contains a nonsingular elliptic curve, especially, $-K_{M}=\Gamma_{s}$, where $\Gamma_{s}$ is the proper transform of $\Gamma_{0}$ in $M$.

Corollary $1 . \Gamma_{s}^{2}=9-s$.

Proof. $K_{M L}^{2}=K_{P^{2}}^{2}-s=9-s$.

Corollary 2. Let $D$ be an irreducible exceptional curve on $M=V\left(\Sigma_{s}\right)$. Then we have either

(i) $D$ is a non-singular rational curve with $D^{2}=-1$ and $D \cdot \Gamma_{s}=1$, or

(ii) $D$ is a non-singular rational curve with $D^{2}=-2$ and $D \cdot \Gamma_{s}=0$.

Proof. Let $g$ be the genus of $D$ and $\delta$ the number of cusps and nodes of $D$. By the adjunction formula,

$$
-D^{2}+D \Gamma_{s}+2 g+2 \delta=2 .
$$

Since $-D^{2}>0$ and $D \cdot \Gamma_{s} \geqq 0$, we have (i) or (ii).

Q.E.D.

Corollary 3. Let $C=\bigcup_{i=1}^{N} C_{i}$ be the union of all the non-singular rational curves $C_{1}, \cdots, C_{N}$ on $M$ with $C_{i}^{2}=-2(1 \leqq i \leqq N)$. Then $\Gamma_{s} \cap$ $C=\phi$.

(3.3) Rational Del Pezzo surfaces. Let $A$ be a rational Del Pezzo surface with the singular points $\left\{x_{i}\right\}=x$ (see (b) in Proposition 9), and 
-: $M \rightarrow A$ be the minimal resolution of the singular points of $A$. We put $C=\pi^{-1}(x)=\bigcup_{i=1}^{i} C_{i}$, where $C_{i}$ 's are the irredudible components of $C$. Then,

Proposition 11 ([6], [9]). There exists a set $\Sigma_{s}$ of points in $\boldsymbol{P}^{2}$ which are in almost general position such that $M=V\left(\Sigma_{s}\right)$.

By Corollaries 2 and 3, we have

Proposition 12 . $-K_{-4}=\Gamma$, where $\Gamma$ is a non-singular elliptic curve in $A$ such that $\Gamma \cap\{x\}=\phi$.

Proposition 13. The following (1), (2) and (3) are equivalent,

(1) $H^{2}(A ; Z) \simeq Z$

(2) $\operatorname{Pic} A \simeq Z\left(-K_{A}\right)$

(3) $C=\bigcup_{i=1}^{s} C_{i}$, where $s=\left|\Sigma_{s}\right|$

Proof. Let us consider the following exact sequences of cohomology (c.f. Brenton [2]):

$$
\begin{aligned}
& \longrightarrow H^{1}(C ; Z) \longrightarrow H^{2}(A ; Z) \longrightarrow H^{2}(M ; Z) \\
& \longrightarrow H^{2}(C ; Z) \longrightarrow H^{3}(A ; Z) \longrightarrow H^{3}(M ; Z) \longrightarrow \\
& 0 \longrightarrow H^{1}\left(A, \mathcal{O}_{A}\right) \longrightarrow H^{1}\left(M, \mathcal{O}_{M}\right) \\
& \longrightarrow H^{0}\left(A, R^{1} \pi^{*} \mathcal{O}_{M}\right) \longrightarrow H^{2}\left(A, \mathcal{O}_{A}\right) \longrightarrow
\end{aligned}
$$

Since the self-intersection number of each irreducible component $C_{\imath}$ of $C$ is equal to -2 , the singular points of $A$ are rational double points, and thus $H^{1}(C ; Z)=0, H^{0}\left(A, R^{1} \pi^{*} \mathcal{O}_{M}\right)=0$. Since $M$ is a rational surface, $H^{1}\left(M, \mathcal{O}_{M}\right)=0=H^{1}(M ; Z)$. Hence $\operatorname{rank} H^{3}(A ; Z)=\operatorname{rank} H^{3}(M ; Z)=$ $\operatorname{rank} H^{1}(M ; Z)=0$. Since $M=V\left(\Sigma_{s}\right), H^{2}(M ; Z) \simeq Z^{s+1}$. We have, by (*), $\operatorname{rank} H^{2}(C ; Z)=s \Longleftrightarrow H^{2}(A ; Z) \simeq Z$. This proves $(1) \Longleftrightarrow(3)$. By $\left(^{* *}\right)$, $H^{1}\left(A, \mathcal{O}_{A}\right)=0$, and further $H^{2}\left(A, \mathcal{O}_{A}\right) \simeq H^{0}\left(A, K_{A}\right) \simeq H^{0}\left(A, \mathcal{O}_{A}(-\Gamma)\right)=0$ by Serre duality. Thus $H^{1}\left(A, \mathcal{O}_{A}^{*}\right) \simeq H^{2}(A ; Z)$, where $\mathcal{O}_{A}^{*}=\mathcal{O}_{A}-\{0\}$. Let $g$ be a generator of $H^{2}(A ; Z)$. Then $\Gamma=k g$ for some integer $k$. Let $\hat{L}$ be an exceptional curve of the first kind in $M$, and put $L=\pi(\hat{L})$. Then,

$$
1=\left(\hat{L} \cdot \Gamma_{s}\right)=(L \cdot \Gamma)=k(L \cdot g),
$$

hence $k=1$. Therefore Pic $A \simeq Z\left(-K_{A}\right) \simeq H^{2}(A ; Z) \simeq Z$. This proves (1) $\Longleftrightarrow(2)$.

Q.E.D.

Corollary 4. $H^{2}(A ; Z) \simeq Z \Rightarrow s \geqq 3$ 
Now, we put $M^{(s)}=V\left(\Sigma_{s}\right), A^{(s)}=A, C^{(s)}=C$ and $C_{i}^{(s)}=C_{i}(1 \leqq i \leqq s)$. Let $E_{s}$ be an exceptional curve of the first kind in $M^{(s)}, M^{(s-1)}=M^{(s)} / E_{s}$ be the contraction of $E_{s}$, and $\pi^{(s)}: M^{(s)} \rightarrow M^{(s-1)}$ the projection. Since $-K_{M(s)}=\Gamma_{s}$ and $E_{s} \cdot \Gamma_{s}=1$, if we set $\Gamma_{s-1}=\pi^{(s)}\left(\Gamma_{s}\right)$, then we have $-K_{M(s-1)}=\Gamma_{s-1}$. Let $C^{(s-1)}=\bigcup_{i}^{N_{s-1}} C_{i}^{(s-i)}$ be the union of all the nonsingular rational curves $C_{i}^{(s-1)}$ with the self-intersection number -2 . We can see that $C^{(s-1)} \subseteq \pi^{(s)}\left(C^{(s)}\right)$. We put $A^{(s-1)}=M^{(s-1)} / C^{(s-1)}$. We have similarly $M^{(s-i)}, C^{(s-i)}=\bigcup_{j}^{N_{s-i}} C_{j}^{(s-i)}, \Gamma^{(s-i)}$ and $A^{(s-i)}(0 \leqq i \leqq s-3)$.

Proposition 14. Pic $A^{(s-i)} \simeq Z\left(-K_{A^{(s-i)}}\right) \Longleftrightarrow N_{s-i}=s-i$

Proof. Since $H^{2}\left(M^{(s-i)} ; Z\right) \simeq Z^{s-i+1}, H^{2}\left(C^{(s-i)} ; Z\right) \simeq Z^{N_{s-i}}$, and rank $H^{3}\left(A^{(s-i)} ; Z\right)=\operatorname{rank} H^{3}\left(M^{(s-i)} ; Z\right)=0$, we have, by a similar exact sequence to $\left.{ }^{*}\right), H^{2}\left(A^{(s-i)} ; Z\right) \simeq Z \Longleftrightarrow N_{s-i}=s-i$.

Q.E.D.

(3.4) Determination of the points of blowing up. Let $\Gamma$ be an elliptic curve, and $P_{0} \in \Gamma$ be a fixed point. We put $\operatorname{Pic}^{0} \Gamma=\{\xi \in \operatorname{Pic} \Gamma ; \operatorname{deg} \xi=0\}$. As a consequence of the Riemann-Roch theorem, the map $P \rightarrow \mathcal{O}\left(P-P_{0}\right)$ induces a bijection between the set of points of and the group $\operatorname{Pic}^{0} \Gamma$. Thus the set of points of $\Gamma$ form a group, with $P_{0}$ as the 0 element, and with addition characterized by $R+Q \equiv R$ if and only if $P+Q \sim R+P_{0}$ as divisors on $\Gamma$. This is the group structure on $\left(\Gamma, P_{0}\right)$. If we embed $\Gamma$ in $\boldsymbol{P}^{2}$ by the linear system $\left|\mathcal{O}\left(3 P_{0}\right)\right|$, then three points $P, Q, R$ of the image are on a line if and only if $P+Q+R \sim 3 P_{0}$, namely, $P+Q+R \equiv 0$ in the group structure (see Hartshorne [8]). Thus, a point $P$ is a point of inflection of $T$ if and only if $3 P \equiv 0$.

Let $q_{2}, q_{3}, \cdots, q_{r}$ be infinitely near a point $q_{1} \in \Gamma_{0}$. Then we set $q_{1}^{1}=$ $\left\{q_{1}\right\}, q_{1}^{2}=\left\{q_{1}, q_{2}\right\}, \cdots, q_{1}^{r}=\left\{q_{1}, \cdots, q_{1}\right\}$. Now, we put $\sigma_{s}=\Sigma_{s}$ - infinitely near points $\}=\left\{P_{1}, \cdots, P_{k}\right\}(k \leqq s)$. We say that a point of $\sigma_{s}$ is an ordinary point of $\Sigma_{s}$. Then,

$$
\Sigma_{s}=P_{1}^{s_{1}} \cup P_{2}^{s_{2}} \cup \cdots \cup P_{k}^{s_{k}},
$$

where $s_{1}+s_{2}+\cdots+s_{k}=s$.

Let $\Gamma_{0}$ be a non-singular elliptic curve through all the points of $\Sigma_{s}$. Then the configuration of $V\left(\Sigma_{s}\right)$ is (not necessarily uniquely) determined by the relation $\left(R_{s}\right)$ among $P_{1}, \cdots, P_{k} \in \Gamma_{0}$ in the group structure of $\Gamma_{0}$.

In the case of $3 \leqq s \leqq 7$, one can get easily the relation $\left(R_{s}\right)$ and is already known but in the case of $s=8$, it is more complicated (see [9], [4]). Let us consider the case of $s=8$ using the theory of elliptic surfaces 
(see Kodaira [12]). Let $\Gamma^{\prime}$ and $\Gamma^{\prime \prime}$ be two non-singular members of the linear system $\left|-K_{M^{(8)}}\right|$. Since $\left(K_{M^{(8)}}\right)^{2}=1$, the intersection of $\Gamma^{\prime}$ and $\Gamma^{\prime \prime}$ is only one point $P$. Let $F$ be a rational function such that $(F)=\Gamma^{\prime}-\Gamma^{\prime \prime}$. Then the point $P$ is a point of indeterminacy of $F$. Let $\pi^{(9)}: M^{(9)} \rightarrow M^{(8)}$ be the blowing up of $M^{(8)}$ with center $P$. Then $\hat{F}=\pi^{(9)-1} \cdot F: M^{(9)} \rightarrow P^{1}$ is a holomorphic mapping and the triple $R=\left(M^{(9)}, \hat{F}, P^{1}\right)$ has a structure of an elliptic surface. Let $C=C^{(8)}$ be as above, and $\hat{C}$ be the proper transformation of $C$. Then $\hat{C} \simeq C$ and $\hat{C}$ is contained in singular fibers of $R$, since $C$ does not intersect non-singular members of $\mid-K_{\left.M^{(8)}\right)}$. The types of singular fibres of elliptic surfaces are classified by Kodaira [12]. In our case, $\hat{C}$ is contained in a fibre of one of the following types (Figure I). Where each vertex is a non-singular rational curve with the self-intersection number -2 , and the number adjacent to the vertex is the multiplicity of $F$. We put $E=\pi^{(9)-1}(P)$. Then $E$ is a section of $R$. Let $C_{0}$ be the irreducible component of a singular fiber of $R$ intersecting $E$. Then $F$ has the multiplicity one on $C_{0}$. Thus the graph $\gamma(C)$ can be obtained by the combination of the following where the dotted vertices represent the exceptional curves of the first kind, of course, they are not components of $C$. In the combination of the graphs in Figure $I^{\prime}$, the number of the vertices must be equal to 8 . Blowing down these exceptional curves of the first kind, we can reduce the case of $s=8$ to that of $3 \leqq s \leqq 7$ (see also Proposition 14), and pick up all the possible types of the graph $\gamma(C)$.
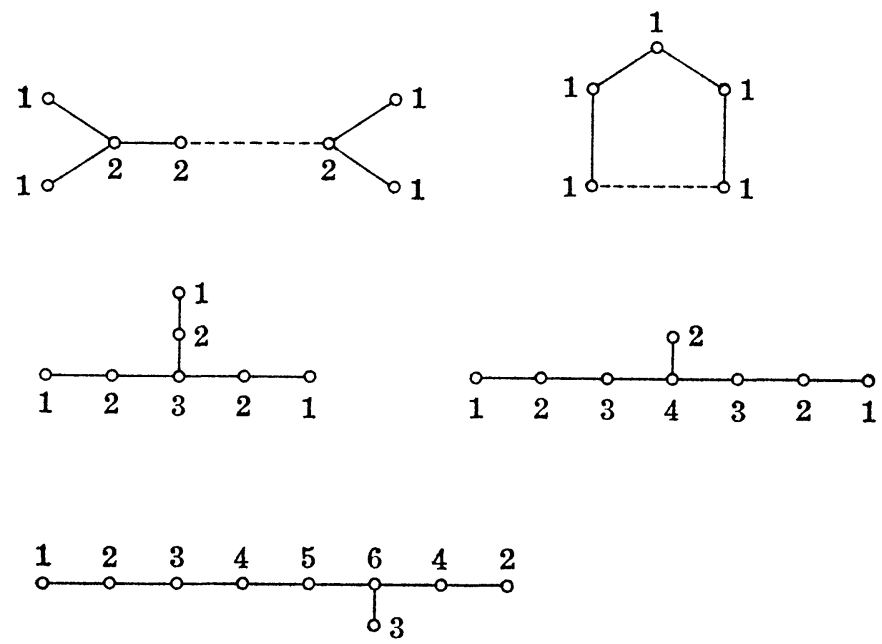

(Figure I) 

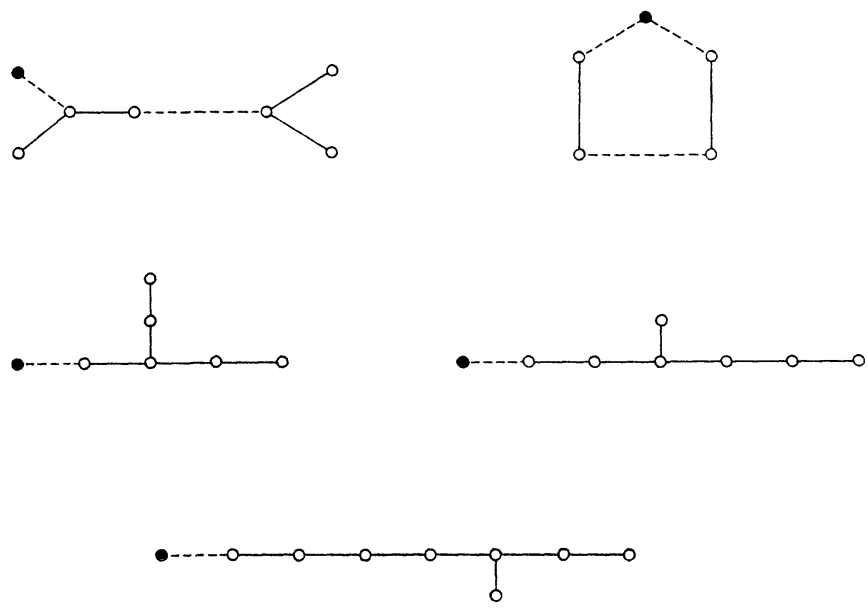

(Figure $I^{\prime}$ )

Thus we have the following

Theorem 2. Let $A$ be a rational singular Del Pezzo surface. Suppose that Pic $A \simeq Z$. Then $K_{A}^{2}=9-s(s \geqq 3)$ and the minimal resolution $M$ of the singularities of $\boldsymbol{A}$ is obtained from $\boldsymbol{P}^{2}$ by blowing up $s$ points $\Sigma_{s}(s \geqq 3)$ on a non-singular elliptic curve $\Gamma_{0}$ which are classified in the Table (II) below, where each $\tilde{D}_{i}$ is a cubic curve in $\boldsymbol{P}^{2}$ with a cusp or node, and the proper transform of $\tilde{D}_{i}$ in $M^{(8)}$ is a non-singular rational curve with the self-intersection number -2 .

Table (II)

\begin{tabular}{|c|c|c|c|}
\hline$s$ & $\begin{array}{c}\text { The type of } \\
\text { Sing } A\end{array}$ & $\Sigma_{s}$ & Relations $\left(R_{s}\right)$ \\
\hline 3 & $A_{2}+A_{1}$ & $P_{1}^{3}$ & $3 P_{1} \equiv 0$ \\
\hline 4 & $A_{4}$ & $P_{1}^{4}$ & $3 P_{1} \equiv 0$ \\
\hline 5 & $D_{5}$ & $P_{1}^{5}$ & $3 P_{1} \equiv 0$ \\
\hline$A_{3}+2 A_{1}$ & $\left(P_{1}^{3}, P_{2}^{2}\right)$ & $\left\{\begin{array}{l}3 P_{1} \equiv 0 \\
2 P_{2}+P_{1} \equiv 0\end{array}\right.$ \\
\hline$E_{6}$ & $P_{1}^{6}$ & $3 P_{1} \equiv 0$ \\
\hline 6 & $\left(P_{1}^{4}, P_{2}^{2}\right)$ & $\left\{\begin{array}{l}3 P_{1} \equiv 0 \\
2 P_{2}+P_{1} \equiv 0\end{array}\right.$ \\
\hline$A_{5}+A_{1}$ & $\left(P_{1}^{3}, P_{2}^{3}\right)$ & $\left\{\begin{array}{l}3 P_{1} \equiv 0 \\
3 P_{2} \equiv 0\end{array}\right.$ \\
\hline $3 A_{2}$ & & \\
\hline
\end{tabular}


Table (II) (Continued)

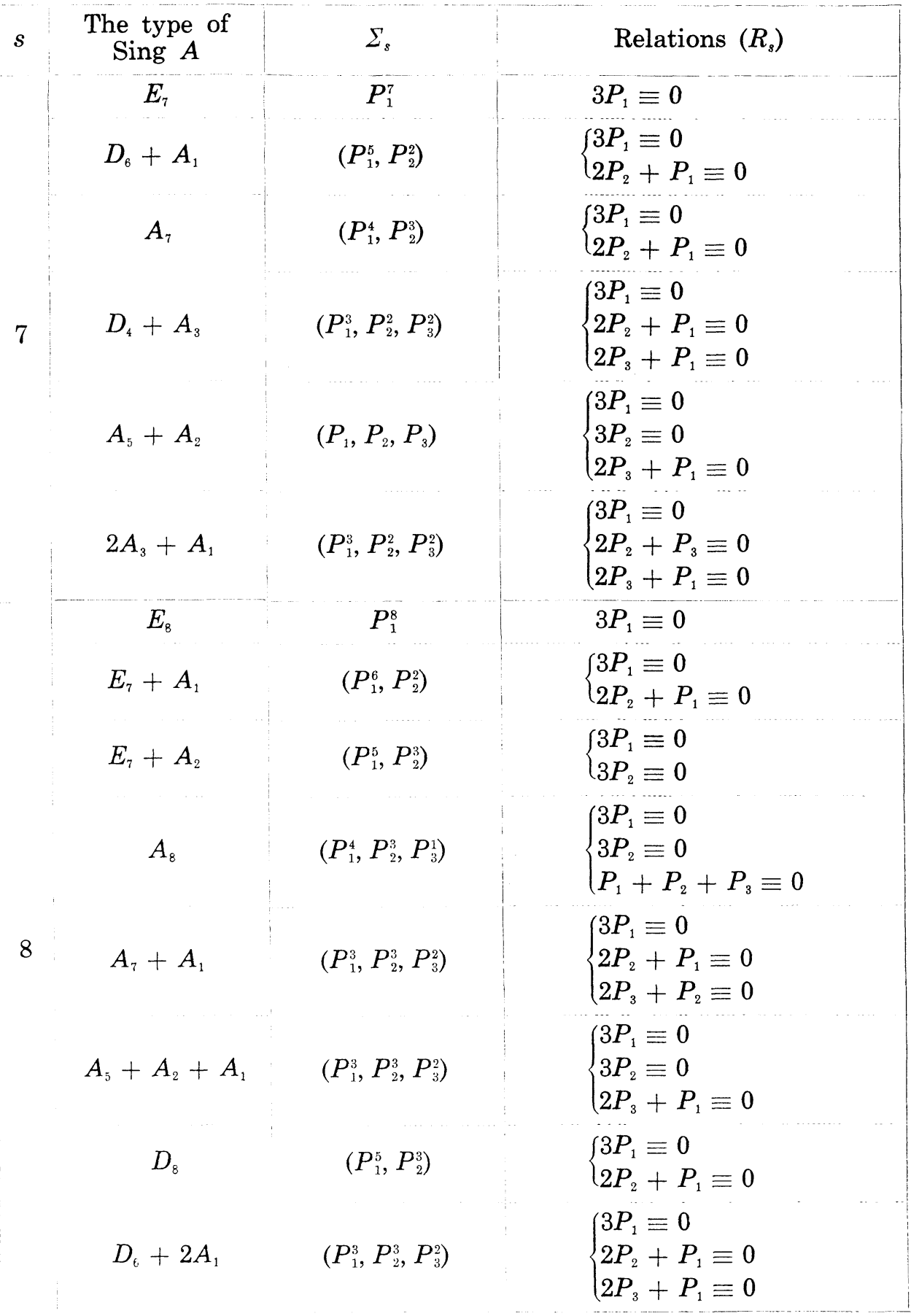


Table (II) (Continued)

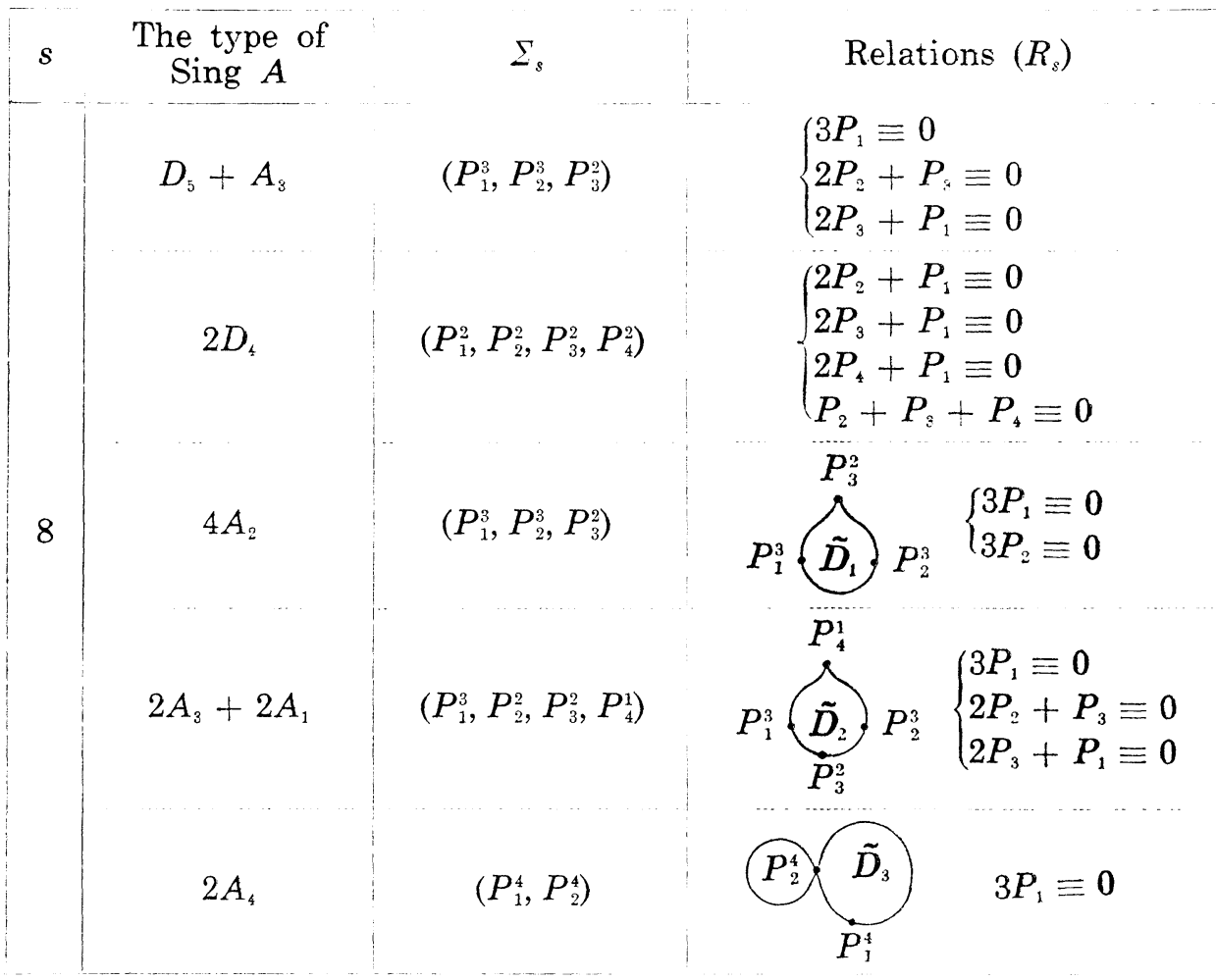

Remark. The position of the points of $\Sigma_{s}$ is not uniquely determined by $M=V\left(\Sigma_{s}\right)$.

\section{$\S 4$. The case of $r=2$}

In the case of $r=2$, we have seen in Section 1 and Section 3 that $K_{X}=-2 A$ and $K_{A}=-\Gamma$, where $\Gamma$ is a non-singular elliptic curve which does not pass through the singular points of $A$. Let $N_{\Gamma}$ be the normal bundle of $\Gamma$ in $A$. Since $\Gamma$ is an elliptic curve, there exists a point $p$ of $\Gamma$ such that $N_{\Gamma}=\mathcal{O}_{\Gamma}(m \cdot p)$, where $m=\operatorname{deg} N_{\Gamma}=\Gamma^{2}\left(=K_{A}^{2}\right)$. By Proposition 8 and Theorem 1, we may assume that $A$ is a rational Del Pezzo surface. Hence $m=9-s(3 \leqq s \leqq 8)$, namely, $1 \leqq m \leqq 6$.

LEMma 2. The Euler number $\chi(X)=4$.

Proof. Since $A$ has only rational double points and it is obtained by $s$ rational curves of $M^{(s)}(3 \leqq s \leqq 8)$, one has $\%(A)=\%\left(\boldsymbol{P}^{2}\right)=3$. Thus $\varkappa(X)=\%(A)+\%\left(C^{3}\right)=4$

Q.E.D. 
(4.1) Non-existence of $(X, A)$ with $m=(A \cdot A \cdot A) \leqq 4$. We can see $\gamma(X) \neq 4$ if $m \leqq 4$, which contradicts Lemma 2 . In fact, if $r=2$ and $m=A^{3}=(A \cdot A \cdot A) \leqq 4$, then by Table 3.5 in $\left[11_{\mathrm{a}}\right]$, we have $\chi(X)=-38$, $-16,-6,0$. Therefore $\chi(X) \neq 4$ (cf. (2.2)).

(4.2) The structure of $(X, A)$ with $m=5$. In this case, by the linear system $\left|\mathcal{O}_{X}(A)\right|, X$ can be realized as a 3 -fold of degree 5 in $\boldsymbol{P}^{6}$ and then $A$ is a hyperplane section.

$\left(1^{\circ}\right)$ First we shall show the following

Proposition 15. There exists unique line $L$ in $P^{6}$ contained in $A \hookrightarrow X$.

Let $\mu: M \rightarrow A$ be the minimal resolution of singular points of $A$, and put $C=\mu^{-1}(a)$, where $\{a\}$ is the set of singular points of $A$. Then $M=$ $V\left(\Sigma_{4}\right)$, where $\Sigma_{4}=\left\{P_{1}, P_{2}, P_{3}, P_{4}\right\}$ are almost general position in $\boldsymbol{P}^{2}$. Let $\Gamma_{0}$ be a non-singular elliptic curve through all the points of $\Sigma_{4}$. Then, by Theorem 2 in Section 3, the point $p_{1}$ is a point of inflection of $\Gamma_{0}$ and the points $P_{i}$ 's $(2 \leqq i \leqq 4)$ are infinitely near the point $P_{1}$. Let

$$
M=V\left(\Sigma_{4}\right) \stackrel{\pi_{4}}{\longrightarrow} V\left(\Sigma_{3}\right) \stackrel{\pi_{3}}{\longrightarrow} V\left(\Sigma_{2}\right) \stackrel{\pi_{2}}{\longrightarrow} V\left(\Sigma_{1}\right) \stackrel{\pi_{1}}{\longrightarrow} \boldsymbol{P}^{2}
$$

be the sequence of the monoidal transformations with center $\Sigma_{j}=\left\{P_{1}, \cdots, P_{j}\right\}$ $(j \leqq 4)$, and put $E_{i}=\pi_{i}^{-1}\left(P_{i}\right)\left(P_{i} \in V\left(\Sigma_{i-1}\right)\right), \hat{L}=\pi_{4}^{-1}\left(P_{4}\right)$ and $\pi=\pi_{1} \circ \pi_{2} \circ \pi_{3} \circ \pi_{4}$. Then we have

$$
\begin{aligned}
\pi^{-1}\left(P_{1}\right) & =\hat{E}_{1} \cup \hat{E}_{2} \cup \hat{E}_{3} \cup \hat{L} \\
C & =\hat{C}_{0} \cup \hat{E}_{1} \cup \hat{E}_{2} \cup \hat{E}_{3}
\end{aligned}
$$

where $\hat{E}_{i}$ is the proper transform of $E_{i}$ in $M$ with the self-intersection number -2 and $\hat{C}_{0}$ is the proper transform of the line tangent to $\Gamma_{0}$ at the point $P_{1}$. Thus the graph $\gamma(C)$ of $C$ is of the form

$$
\gamma(C): \begin{array}{cccc}
-2 & -2 & -2 & -2 \\
\circ & \circ & 0 & 0
\end{array}
$$

and the configuration of $C$ and $\hat{L}$ look like Figure 1. We put $\Gamma=\mu\left(\Gamma_{4}\right)$ and $L=\mu(\hat{L})$. Then, Pic $A \simeq Z\left(-K_{A}\right) \simeq Z([\Gamma])$ by Proposition 9 in Section 3. Since $(L \cdot T)_{A}=\left(\hat{L} \cdot \Gamma_{4}\right)_{M}=1$, we have $(L \cdot A)_{X}=1$. Hence, $L$ is a line of $\boldsymbol{P}^{6}$ contained in $A \subset X . L$ is the unique line contained in $A$. In fact, let $E \neq L$ be a line in $A$, and $E^{\prime}$ the proper transform of $E$ in $M$. Then $\left(E^{\prime} \cdot \Gamma_{4}\right)_{M}=(E \cdot \Gamma)_{A}=1$, hence $E^{\prime}$ is an exceptional curve of the first kind. 
We put $D=\pi\left(E^{\prime}\right)$ and $d=\operatorname{deg} D \geqq 1$. Let $\nu_{i}$ be the multiplicity of $D$ at $p_{i}$, where $\nu_{i}=0$ means that $p_{i} \notin D$. By Hurwitz formula,

$$
(d-1)(d-2)-\sum_{i=1}^{4} \frac{\nu_{i}\left(\nu_{i}-1\right)}{2}=0
$$

that is, $d^{2}-3 d+2=\sum_{i=1}^{4} \nu_{i}^{2}-\sum_{i=1}^{4} \nu_{i}$. Since $-1=E^{\prime 2}=d^{2}-\sum_{i=1}^{4} \nu_{i}^{2}$, we have $\sum_{i=1}^{4} \nu_{i}^{2}=d^{2}+1$ and $\sum_{i=1}^{4} \nu_{i}=3 d-1$. By Schwarz' inequality, $4\left(\sum_{i=1}^{4} \nu_{i}^{2}\right) \geqq\left(\sum_{i=1}^{4} \nu_{i}\right)^{2}$, namely, $4\left(d^{2}+1\right) \geqq(3 d-1)^{2}$. Thus we have $5 d^{2}-$ $6 d-3 \leqq 0$ Hence $d=1, \nu_{1}=1, \nu_{2}=1, \nu_{3}=0, \nu_{4}=0$. Then $\left\{p_{1}, p_{2}\right\}$ are on $D$. Therefore we have $C_{0}=D$. This is a contradiction.

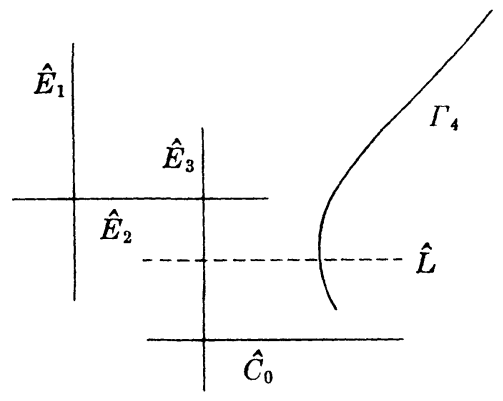

Figure 1 .

$\left(2^{\circ}\right)$ Next, we shall show the following

Proposition 16. Let $\Phi: X \rightarrow P^{4}$ be the projection from the line $L$ to $\boldsymbol{P}^{4}$. We put $W=\Phi(X)$ and $V=\Phi(A)$. Then,

(1) $W$ is a non-singular quadric hypersurface in $P^{i}$ and $V$ is a tangent hyperplane section of $W$.

(2) Let $\sigma_{L}: X^{\prime} \rightarrow X$ be the monoidal transformation with center $L$. Then we have the following diagram:

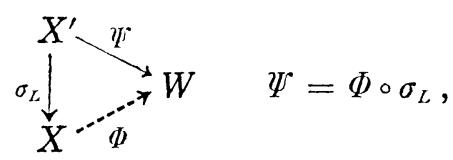

where $\Psi: X^{\prime} \rightarrow W$ is a birational morphism. Further, we have

(i) The exceptional set $E$ of $\Phi$ is an irreducible ruled variety of dimension 2 swept out by lines which meat the line $L$,

(ii) the image $Y=\Phi(E)$ is a non-singular rational twisted cubic curve contained in $\Psi\left(\sigma_{L}^{-1}(L)\right)=V_{0} \cong \boldsymbol{Q}_{0}^{2}$. 
(iii) the birational morphism $\Psi: X^{\prime} \rightarrow W$ is the monoidal transformation of $W$ with center $Y$.

We shall study the linear systems $\left|\mathcal{O}_{A}(T-L)\right|$ and $\left|\mathcal{O}_{X}(A-L)\right|$. Since $A \subset \boldsymbol{P}^{5}$ and $\Gamma$ is a hyperplane section of $A$, we have $\operatorname{dim} H^{0}\left(A ; \mathcal{O}_{A}(I-L)\right)$ =4. Let $\left\{f_{i}\right\}(2 \leqq i \leqq 6)$ be a basis of $H^{0}\left(\Gamma ; \mathcal{O}_{\Gamma}(5 p)\right)$ such that $f_{3} / f_{2}=\mathfrak{p}$, $f_{4} / f_{2}=\mathfrak{p}^{\prime}, f_{5} / f_{2}=\mathfrak{p}^{2}, f_{6} / f_{2}=\mathfrak{p} \cdot \mathfrak{p}^{\prime}$. Let $\phi_{i}$ be a section of $H^{0}\left(A ; \mathcal{O}_{A}(\Gamma)\right)$ such that $\phi_{i} \mid A=f_{i}$. Then $\phi_{2}, \phi_{3}, \phi_{4}, \phi_{5} \in H^{0}\left(A ; \mathcal{O}_{A}(\Gamma-L)\right)$. Let $\hat{\phi}_{i}$ be the proper transform of $\dot{\phi}_{i}$ in $M$. Then we have

$$
\begin{aligned}
& (\hat{\dot{\partial}})=5 \hat{L}+2 \hat{E}_{1}+4 \hat{E}_{2}+6 \hat{E}_{3}+3 \hat{C}_{0} \\
& \left(\hat{\zeta}_{3}\right)=3 \hat{L}+2 \hat{E}_{1}+3 \hat{E}_{2}+4 \hat{E}_{3}+2 \hat{C}_{0}+\hat{C}_{1} \\
& (\dot{\dot{\zeta}})=2 \hat{L}+\hat{E}_{1}+2 \hat{E}_{2}+3 \hat{E}_{3}+2 \hat{C}_{0}+\hat{C}_{2} \\
& \left(\hat{\dot{G}}_{5}\right)=\hat{L}+\hat{E}_{1}+2 \hat{E}_{2}+2 \hat{E}_{3}+\hat{C}_{0}+2 \hat{C}_{1} \text {, }
\end{aligned}
$$

where $C_{1}$ is a line intersecting transversally $\Gamma_{0}$ at the point $p_{1}, C_{2}$ is a line with $C_{2} \cap \Sigma_{4}=\phi$, and $\hat{C}_{i}$ 's $(1 \leqq i \leqq 2)$ are the proper transforms of $C_{i}$ in $M$. Let $\equiv H^{\prime}\left(\boldsymbol{P}^{2} ; \mathcal{O}\left(\Gamma_{0}\right)\right)$ be a section such that $\psi_{i} \cdot \pi=\hat{o}_{i}$. Then we have

$$
\begin{aligned}
& \left(\psi_{2}\right)=3 C_{0} \\
& \left(\psi_{3}\right)=2 C_{0}+C_{1} \\
& \left(\psi_{4}\right)=2 C_{0}+C_{2} \\
& \left(\psi_{5}\right)=C_{0}+2 C_{1} .
\end{aligned}
$$

Let $\left(X_{0}: X_{1}: X_{2}\right)$ be the homogeneous coordinate system in $\boldsymbol{P}^{2}$. We put the defining equation of $\Gamma_{0}: X_{0} X_{2}^{2}=4 X_{1}^{3}+g_{2} X_{1} X_{0}^{2}+g_{3} X_{0}^{3}$. Then the birational mapping $\psi=\left(r_{2}: \gamma_{3}: \psi_{4}: \psi_{5}\right): \boldsymbol{P}^{2} \rightarrow \boldsymbol{P}^{3}$ is given by

$$
\left\{\begin{array}{l}
w_{0}=X_{0}^{3} \\
w_{1}=X_{0}^{2} X_{1} \\
w_{2}=X_{0}^{2} X_{2} \\
w_{3}=X_{0} X_{1}^{2}
\end{array}\right.
$$

where $\left(w_{0}: \cdots: u_{3}\right)$ is the homogeneous coordinate system of $\boldsymbol{P}^{3}$. The indeterminant point of $\psi$ is the point $(0: 0: 1) \in \boldsymbol{P}^{2}$, and $V=\psi\left(\boldsymbol{P}^{2}\right)=$ $\left\{w_{0} w_{3}=w_{1}^{2}\right\} \leftrightharpoons \boldsymbol{P}^{3}$. Then $\psi^{\prime}=\left(\pi_{1} \circ \pi_{2}\right)^{-1} \psi^{\prime}: V\left(\Sigma_{2}\right) \rightarrow V \longrightarrow \boldsymbol{P}^{3}$ is a birational morphism (the resolution of indeterminant points of $\psi$ ). Therefore, $\phi=\left(\phi_{2}: \phi_{3}: \phi_{1}: \phi_{3}\right): A \rightarrow \boldsymbol{P}^{3}$ is a birational mapping, $L$ is the set of indeterminant points of $\dot{\phi}$, and $\phi(A)=V$. Thus we have the diagram: 


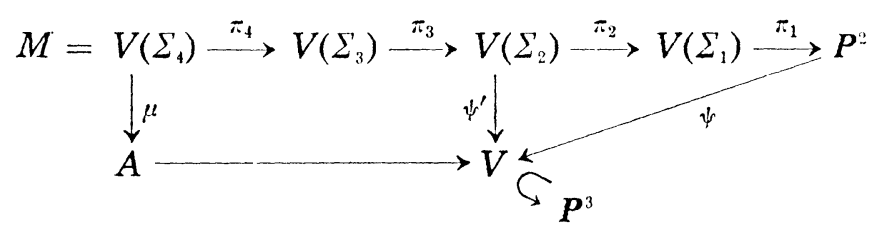

Let $\Phi_{i}(2 \leqq i \leqq 5)$ be sections of $H^{0}\left(X ; \mathcal{O}_{X}(A-L)\right)$ such that $\left.\Phi_{i}\right|_{A}=\phi_{i}$ and $\left(\Phi_{0}\right)=A$. Since $\operatorname{dim} H^{0}\left(X ; \mathcal{O}_{X}(A-L)\right)=5,\left\{\Phi_{0}, \Phi_{2}, \cdots, \Phi_{5}\right\}$ is a basis of $H^{0}\left(X ; \mathcal{O}_{X}(A-L)\right)$. Let us consider the rational mapping $\Phi=\left(\Phi_{0}: \Phi_{2}\right.$ : , $\left.\cdots,: \Phi_{5}\right): X \rightarrow P^{4}$ defined by the linear system $\left|\mathcal{O}_{X}(A-L)\right|$. By the construction, we find that $\left.\Phi\right|_{A}=\phi$. Let $\sigma: X^{\prime} \rightarrow X$ be the blowing up of $X$ with center $L$, and $A^{\prime}$ be the proper transform of $A$ in $X^{\prime}$, and set $L^{\prime}=$ $\sigma^{-1}(L)$. Then $L^{\prime}$ is a ruled surface.

Lemma 6 (see [11]). Let $A(X)$ (resp. $A\left(X^{\prime}\right)$ ) be the Chow ring of $X$ (resp. $X^{\prime}$ ). Let $\ell$ be the class of a fibre of $L^{\prime}$. Then,

(i) $A\left(X^{\prime}\right) \simeq \sigma^{*} A(X)+Z L^{\prime}+Z \ell$ as additive group, with $\sigma_{*}\left(L^{\prime}\right)=$ $\sigma_{*}(\ell)=0$, and $\sigma_{*} \sigma^{*} A(X)=A(X)$.

(ii) Let $c_{1}(X)$ be the first Chern class of $X, N_{L / X}$ normal bundle of $L$ in $X$, and $c_{1}\left(N_{L / X}\right)$ its first Chern class. Then,

$\left(L^{\prime}\right)^{2}=-\sigma^{*}(L)+c_{1}\left(N_{L / X}\right)$

$\left(L^{\prime}\right)^{3}=-c_{1}\left(N_{L / X}\right)$,

$\left(L^{\prime} \cdot \ell\right)=-1$,

$\left(L^{\prime} \cdot \sigma^{*}(D)\right)=(L \cdot D) \cdot \ell,\left(\ell \cdot \sigma^{*}(D)\right)=0$ for all $D \in A^{1}(X)$,

$\left(L^{\prime} \cdot \sigma^{*}(C)\right)=\left(\ell \cdot \sigma^{*}(C)\right)$ for all $C \in A^{2}(X)$,

where we denote the $i$-th component of the Chow ring $A(X)$ by $A^{i}(X)$, graded by codimension.

Furthermore, we have

$$
c_{1}\left(N_{L / X}\right)+2-2 g(L)=\left(c_{1}(X) \cdot L\right),
$$

where $g(L)$ is the genus of $L$.

The linear system $\left|\mathcal{O}_{X^{\prime}}\left(\sigma^{*} A-L^{\prime}\right)\right|=\left|\mathcal{O}_{X^{\prime}}\left(A^{\prime}\right)\right|$ is without fixed components and base points. Thus the rational mapping $\Psi: X^{\prime} \rightarrow \boldsymbol{P}^{4}$ defined by the linear system $\left|\mathcal{O}_{X^{\prime}}\left(A^{\prime}\right)\right|$ is a morphism. We put $W=\Psi\left(X^{\prime}\right)$. Since $c_{1}\left(N_{L / X}\right)=2(A \cdot L)-2=0$, we have, by Lemma $6,\left(A^{\prime}\right)^{3}=\left(\sigma^{*} A-L\right)^{3}=$ $A^{3}-3\left(A \cdot L^{\prime} \cdot L^{\prime}\right)-\left(L^{\prime}\right)^{3}=2$. Hence, deg $W=2$. Further, we can see that $\Psi$ is a birational morphism of $X$ onto $W$, and thus, the restriction $\left.\Psi\right|_{1^{\prime}}$ : $A^{\prime} \rightarrow V$ is a birational morphism since every irreducible quadric is normal. 
We put $\Psi\left(L^{\prime}\right)=V_{0}$. Since $\left(L^{\prime} \cdot A^{\prime} \cdot A^{\prime}\right)_{X^{\prime}}=L^{\prime}\left(\sigma^{*} A-L^{\prime}\right)^{2}=2, \operatorname{deg} V_{0}=2$. Thus $V_{0}$ and $V$ are hyperplane sections of the quadric $W \longrightarrow \boldsymbol{P}^{4}$.

Let $\left\{a_{0}\right\}$ be a singular point of $A$. Since the graph of $C$ looks like the following

$$
\gamma(C): \begin{array}{cccc}
-2 & -2 & -2 & -2 \\
\circ & 0 & 0 & \circ
\end{array}
$$

if we choose the coordinates $x, y, z$ is a neighbourhood $\Delta$ of $\left\{a_{0}\right\}$ in $X$, then we may assume that

$$
\Delta \cap A=\left\{z^{5}=x y\right\}, \quad \text { where } a_{0}=(0,0,0) .
$$

The minimal resolution of the singularity of $\Delta \cap A$ is the manifold $M(2,2,2,2)$ (see Laufer [14]). Then we have

$$
L \cap \Delta=\left\{x=z^{2}, y=z^{3}\right\} .
$$

By the coordinate transformation

$$
\begin{aligned}
& \left\{\begin{array}{l}
x^{\prime}=z \\
y^{\prime}=x-z^{2} \\
z^{\prime}=y-z^{3},
\end{array}\right. \\
& A \cap \Delta=\left\{y z+y x^{3}+x^{2} z=0\right\}, \\
& L \cap \Delta=\{y=z=0\} \text {. }
\end{aligned}
$$

Let $\sigma_{L}: \tilde{J} \rightarrow \Delta$ be the monoidal transformation of $\Delta$ with center $L \cap \Delta$, and set $\tilde{\mathcal{J}}=\tilde{\mathcal{J}}_{1}(u, v, w) \cup \tilde{\mathcal{L}}_{2}\left(u^{\prime}, v^{\prime}, w^{\prime}\right)$ where

$$
\sigma_{L}\left\{\begin{array}{l}
x=u=u^{\prime} \\
y=v w=v^{\prime} \\
z=w=v^{\prime} w^{\prime} \quad \text { (see Griffiths-Harris [p. 603, 7]). }
\end{array}\right.
$$

By a direct calculation, the proper transform $A^{\prime}$ of $A \cap \Delta$ in $\tilde{\Delta}$ has two rational double points as singular points. Further, we find that $A^{\prime}$ is obtained from $M$ by blowing down the exceptional curves $\hat{E}_{1}$ and $\hat{E}_{3} \cup \hat{C}_{0}$. Let $\tau: M \rightarrow A^{\prime}$ be the projection. Then $\sigma_{L}^{-1}\left(a_{0}\right)=\tau\left(\hat{E}_{2}\right), \Psi(\tau(\hat{L}))=\left\{v_{0}\right\}$ is a regular point of $V, \Psi\left(\tau\left(E_{1}\right)\right)=\{v\}$ is a vertex of $V \simeq \boldsymbol{Q}_{0}^{2}$, and $\Psi\left(\tau\left(E_{2}\right)\right)=\ell_{0}$ is a generating line in $V$ passing through the points $v$ and $v_{0}$.

LEMMA 7. $L^{\prime} \simeq F_{2}$ and $V_{0} \simeq \boldsymbol{Q}_{0}^{2} \longrightarrow \boldsymbol{P}^{3}$.

Proof. By Proposition (5.2) of [11], $L^{\prime}$ is isomorphic to $\boldsymbol{F}_{2}$ or $\boldsymbol{P}^{1} \times \boldsymbol{P}^{1}$. Suppose that $L^{\prime} \simeq \boldsymbol{P}^{1} \times \boldsymbol{P}^{1}$. The curve $\tau(\hat{L})$ is considered as a curve in $L^{\prime}$. Since $\Psi(\tau(\hat{L}))=\left\{v_{0}\right\}, \tau(\hat{L})$ is an exceptional curve in $L^{\prime} \simeq \boldsymbol{P}^{1} \times \boldsymbol{P}^{1}$. 
This is a contradiction, Therefore $L^{\prime} \simeq \boldsymbol{F}_{2}$, and thus $V_{0}=\Psi\left(L^{\prime}\right) \simeq \boldsymbol{Q}_{0}^{2} \longrightarrow \boldsymbol{P}^{3}$, since any quadric hypersurface in $\boldsymbol{P}^{3}$ is isomorphic to $\boldsymbol{Q}_{0}^{2}$ or $\boldsymbol{P}^{1} \times \boldsymbol{P}^{1}$, if it is irreducible.

Q.E.D.

Lemma 8. Let $V \longrightarrow P^{4}$ be a singular quadric hypersurface and $H a$ hyperplane in $\boldsymbol{P}^{4}$ such that $H \cap V \simeq \boldsymbol{Q}_{0}^{2}$. Let $v_{0}$ be the vertex of $H \cap V$, and Sing $V$ the singular locus of $V$. Then $H \cap \operatorname{Sing} V=\left\{v_{0}\right\}$, and $\left(H \cap V-\left\{v_{0}\right\}\right) \cap$ Sing $V=\phi$.

Proof. Since $V$ is an irreducible singular quadric hypersurface in $\boldsymbol{P}^{4}$, Sing $V=\left\{v_{0}\right\}$ (a point) or $L$ (a line in $P^{4}$ ). In the case of $\operatorname{Sing} V=L$, $H \cap V \simeq \boldsymbol{Q}_{0}^{2}$ if and only if $L$ does not contained in $H$. This proves the Lemma.

Q.E.D.

LEMma 8. $W=\Psi\left(X^{\prime}\right)$ is non-singular.

Proof. Suppose that $W$ is a singular quadric hypersurface. The point $\left\{v_{0}\right\}=\Psi(\tau(L))$ is a regular point of $V$ and a singular point of $V_{0}$. Since both $V$ and $V_{0}$ are tangent hyperplane sections of $W$, this contradicts the above lemma.

Q.E.D.

Let $Y^{\prime}$ be the exceptional set of $\Psi$, and set $Y=\Psi\left(Y^{\prime}\right) \hookrightarrow W, E=$ $\sigma\left(Y^{\prime}\right), D^{\prime}=Y^{\prime} \cap L^{\prime}$. Then $X^{\prime}-Y^{\prime} \simeq W-Y$.

Lemma 9. $X-E$ is isomorphic to $C^{3}$.

Proof. We shall show that $Y$ is contained in $V_{0}$. In fact, assume that $Y \sqsubset V_{0}$. Then there exists a point $y$ of $Y$ such that $y \notin V_{0}$. Hence $\Psi^{-1}(y) \cap$ $L^{\prime}=\phi$, namely, $\sigma\left(\Psi^{-1}(y)\right) \cap L=\phi$. Let us denote the 2-dimensional projective plane in $\boldsymbol{P}^{6}$ spanned by $\{y\}$ and $L$ by $\{y\} \cup L$, and set $E_{y}=X \cap(\{y\} \cup L)$. Then $\Phi\left(E_{y}\right)=y$. Since $\sigma\left(\Psi^{-1}(y)\right)$ is a curve of $X$ and $\sigma\left(\Psi^{-1}(y)\right) \subset\{y\} \cup L$ $\simeq \boldsymbol{P}^{2}$, we have $\sigma\left(\Psi^{-1}(y)\right) \cap L=\phi$. This is a contradiction. Therefore $Y \subset V_{0}$. Further, $X^{\prime}-\Psi^{-1}\left(V_{0}\right) \simeq W-V_{0} \simeq X^{\prime}-\left(L^{\prime} \cup Y^{\prime}\right)$. Since $W-V_{0} \simeq C^{3}$ and $\sigma\left(L^{\prime}\right)=L \subset E$, we have $X-E \simeq C^{3}$.

Q.E.D.

Lemma 10. E is a ruled variety of dimension 2, swept out by lines which meet the line $L$.

Proof (compare [11]). Since $X-E \simeq C^{3}$ by Lemma 9, one can sae that $E$ is irreducible by the exact sequence of the relative cohomology corresponding the pair $(X, E)$. Given an arbitrary point on $X$, there is a line $\longrightarrow X$ through it [11]. Thus there is a surface swept out by lines 
which meet the line $L$. Since it is contained in irreducible $E$, it must coincide with $E$.

Q.E.D.

Lemma 11. $Y^{\prime}$ is linearly equivalent to $\sigma^{*} A-2 L^{\prime}$.

Proof. Since $X-E \simeq C^{3}$, we have Pic $X \simeq Z[E]$. On the other hand, since $\operatorname{Pic} X \simeq Z[A], A$ is linearly equivalent to $E$. For simplicity, we write $A=E$. Then $Y^{\prime}=\sigma^{*} A-k L^{\prime}$ for some integer $k>0$. Since $Y^{\prime}$ is a degenerate set, we have $Y^{\prime} \cdot\left(\sigma^{*} A-L^{\prime}\right)^{2}=0$. Using Lemma 6 , we have $k=2$.

Q.E.D.

LeMma $12 . \quad Y$ is a non-singular rational curve of degree 3.

Proof. By the adjunction formula and Lemma 6, we have

$$
\begin{aligned}
& K_{X^{\prime}}=-2 \sigma^{*} A+L^{\prime} \\
& K_{L^{\prime}}=-2 \ell+2\left(L^{\prime}\right)^{2},
\end{aligned}
$$

where $\ell$ is the class of a fibre of $L^{\prime}$. On the other hand, since $L^{\prime} \simeq \boldsymbol{F}_{2}$ by Lemma 7 , we have $K_{L^{\prime}}=-4 \ell-2 s_{0}$, where $s_{0}=\tau(L)$ is the zero section of $L^{\prime}$. Hence $\left(L^{\prime}\right)^{2}=-\ell-s_{0}$. Let $Y^{*}$ be the proper transform of $Y$ in $L^{\prime}$, Since $D^{\prime}=L^{\prime} \cdot\left(\sigma^{*} A-2 L^{\prime}\right)=\ell+2\left(L^{\prime}\right)^{2}=3 \ell+2 s_{0}$ and $s_{0}$ is contained in $L^{\prime}$, we have $Y^{*}=3 \ell+s_{0}$. Let $s_{\infty}$ be a section of $L^{\prime}$ with $s_{\infty}^{2}=2$. Then $Y^{*} \cdot s_{\infty}=\left(3 \ell+s_{0}\right) \cdot s_{\infty}=3$ and $\ell \cdot Y^{*}=1$. Therefore $Y$ is a rational curve of degree $3 . \quad Y$ is non-singular, in fact, if $Y$ has singular points, then $Y$ is contained in a complex projective plane $\boldsymbol{P}^{2}$. Thus $Y$ is contained in the intersection of the plane and the quadric $V_{0}$. Since $\operatorname{deg} Y=3$, this a contradiction.

Q.E.D.

Since $W, Y$ are non-singular, By Lemmas 8, 12, and since $X^{\prime}-Y^{\prime} \simeq$ $X-Y$, we have, by Griffith-Harris [7], that the birational morphism $\Psi$ : $X^{\prime} \rightarrow W$ is the blowing up of $W$ with center $Y$. Therefore, the proof of Proposition 16 is complete.

(4.3) The construction of $(X, A)$ with $m=5$. Let $W$ be a non-singular quadric hypersurface in $\boldsymbol{P}^{4}, \ell$ be an arbitrary line contained in $W$, and $v, v_{0}$ $\left(v \neq v_{0}\right)$ be arbitrary points of $\ell$. Let $V$ (resp. $V_{0}$ ) be a tangent hyperplane section of $W$ at the point $v$ (resp. $v_{0}$ ), and $Y$ be a non-singular rational twisted cubic curve contained in $V_{0}$. Then, $V \cdot V_{0}=2 \ell$ and $Y$ passes through the vertex $v_{0}$ of $V_{0}$. The pair $(W, Y)$ is uniquely determined up to projective equivalence (see p. 513 in [11]). 
Let $\left(w_{0}: w_{1}: w_{2}: w_{3}: w_{4}\right)$ be the homogeneous coordinate system of $\boldsymbol{P}^{4}$. Then we may assume that

$$
\begin{aligned}
& W=\left\{w_{2}^{2}=w_{0} w_{1}+w_{3} w_{4}\right\} \\
& V=\left\{w_{1}=0\right\} \cap W \\
& V_{0}=\left\{w_{4}=0\right\} \cap W \\
& Y=\left\{w_{4}=0, w_{0} w_{2}=w_{1} w_{3}, w_{0}^{2}=w_{2} w_{3}\right\} \cap W .
\end{aligned}
$$

Then, $\ell=\left\{w_{1}=w_{2}=w_{4}=0\right\}, v=(1: 0: 0: 0: 0), v_{0}=(0: 0: 0: 1: 0)$. Let $\Psi:$ $X^{\prime} \rightarrow W$ be the blowing up of $W$ with center $Y$, and set $Y^{\prime}=\Psi^{*-1}(Y)$. Let $L^{\prime}$ be the proper transform of $V_{0}$, and $A^{\prime}$ the proper transform of $V$.

Lemma 13. $L^{\prime}$ is isomorphic to $\boldsymbol{F}_{2}$

Proof. Let us first show that $L^{\prime}$ is non-singular. In the affine part $\left\{w_{3}=1\right\} \simeq C^{4}\left(w_{0}, w_{1}, w_{2}, w_{4}\right)$, we have

$$
\begin{aligned}
& \stackrel{\circ}{W} \simeq\left\{w_{2}^{2}=w_{0} w_{1}+w_{4}\right\}=\longrightarrow C^{4} \\
& \stackrel{\circ}{V} \simeq\left\{w_{1}=0\right\} \cap \dot{W} \\
& \stackrel{\circ}{Y} \simeq\left\{w_{1}=0, w_{1}=w_{0}^{3}, w_{0}=w_{2}^{2}\right\} \\
& \stackrel{\circ}{\ell}_{0} \simeq\left\{w_{1}=w_{2}=w_{4}=0\right\} .
\end{aligned}
$$

We define a one to one regular map $\alpha: C^{3}(x, y, z) \rightarrow C^{1}\left(w_{1}, w_{1}, w_{2}, w_{4}\right)$ by $\alpha$ : $w_{0}=x, w_{1}=y, w_{2}=z, w_{4}=z^{2}-x y$. Then we have

$$
\left\{\begin{array}{l}
\alpha^{-1}(\stackrel{\circ}{W}) \simeq C^{3} \\
\alpha^{-1}\left(\stackrel{\circ}{V_{0}}\right) \simeq\left\{z^{2}=x y\right\} \\
\alpha^{-1}(\stackrel{\circ}{V}) \simeq\{y=0\} \\
\alpha^{-1}(\stackrel{\circ}{Y})=\left\{z=x^{2}, y=x^{5}\right\} \\
\alpha^{-1}\left(\stackrel{\circ}{\ell_{0}}\right)=\{y=z=0\} .
\end{array}\right.
$$

By the coordinate transformation: $u=x, v=y-x^{3}, w=z-x^{2}$, we may assume that in the affine part,

$$
\begin{aligned}
& \stackrel{\circ}{W} \simeq C^{3} \\
& \stackrel{\circ}{V}_{0}=\left\{w^{2}+2 w u^{2}=u v\right\} \\
& \stackrel{\circ}{V}=\left\{v+u^{3}=0\right\} \\
& \stackrel{\circ}{Y}=\{v=w=0\} .
\end{aligned}
$$

Let $\Psi: C_{\dot{Y}}^{3} \rightarrow C^{3}$ be the blowing up of $C^{3}$ with center $\dot{Y}$, and set $C_{\mathrm{I}^{*}}^{3}=\Omega\left(z_{1}, z_{2}, z_{3}\right) \cup \Omega^{\prime}\left(z_{1}^{\prime}, z_{2}^{\prime}, z_{3}^{\prime}\right)$. Then $\Psi$ is given by 


$$
\Psi:\left\{\begin{array}{l}
u=z_{1}=z_{1}^{\prime} \\
v=z_{2} z_{3}=z_{2}^{\prime} \\
w=z_{3}=z_{2}^{\prime} z_{3}^{\prime}
\end{array}\right.
$$

and we have

$$
\begin{aligned}
& L^{\prime} \cap \Omega=\left\{z_{3}+2 z_{1}^{2}=z_{1} z_{2}\right\} \\
& L^{\prime} \cap \Omega^{\prime}=\left\{z_{1}^{\prime}=z_{2}^{\prime} z_{3}^{\prime}+2 z_{3}^{\prime} z_{2}^{\prime 2}\right\} .
\end{aligned}
$$

Therefore $L^{\prime}$ is non-singular. Since the minimal resolution of a singularity of $V_{0}$ is isomorphic to $F_{2}$ and $b_{2}\left(L^{\prime}\right)=2$, we find that $L^{\prime}$ is isomorphic to $F_{2}$ and $\Psi^{-1}\left(v_{0}\right)$ is the zero section of $L^{\prime}$.

Q.E.D.

Lemma 14. $A^{\prime}$ is a normal Gorenstein surface with two rational double points $\left\{a_{0}^{\prime}, a_{1}^{\prime}\right\}$ as singularities. The graph of the minimal resolution of the singularity $\left\{a_{0}^{\prime}\right\}$ (resp. $\left\{a_{1}^{\prime}\right\}$ ) looks like

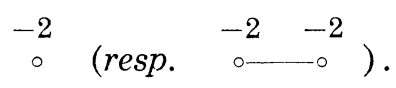

Proof. Since $Y$ does not pass through the vertex $v$ of $V, A^{\prime}$ has a singularity with the graph of the form ${ }^{-2}$. Let $\Omega, \Omega^{\prime}$ be as in Lemma 13. Then we have

$$
\begin{aligned}
& \Omega \cap A^{\prime}=\left\{z_{3}+z_{1}^{3}=0\right\} \\
& \Omega^{\prime} \cap A^{\prime}=\left\{z_{2}^{\prime} z_{3}^{\prime}+z_{1}^{\prime 3}\right\}=0 .
\end{aligned}
$$

Therefore $A^{\prime}$ has a singularity in $\Omega^{\prime}$ with the graph of the form

$$
\begin{array}{cc}
-2 & -2 \\
0 & -0
\end{array}
$$

LEMMA 15. Let $\ell_{0}^{\prime}$ be the proper transform of $\ell_{0}$. Then the singularities $a_{0}^{\prime}$ and $a_{1}^{\prime}$ are contained in $\ell_{0}^{\prime}$.

Proof. We have

$$
\Psi^{-1}\left(\ell_{0}\right) \cap \Omega=\left\{z_{1}=z_{3}=0\right\} \cup\left\{z_{2}=z_{1}, z_{3}+z_{1}^{2}=0\right\} .
$$

Since $\Psi\left(z_{1}=z_{2}=0\right)=(0,0,0)$, we have that

$$
s_{0} \cap \Omega=\left\{z_{1}=z_{2}=0\right\},
$$

where $s_{0}=\Psi^{-1}\left(v_{0}\right)$, and that

$$
\ell_{1)}^{\prime} \cap \Omega=\left\{z_{1}=z_{2}, z_{3}+z_{1}^{2}=0\right\} \ni(0,0,0)=a_{1}^{\prime} . \quad \text { Q.E.D. }
$$


Lemma 16. $L^{\prime}$ and $A^{\prime}$ intersect transversally on $s_{0}$.

Proof. Let us consider $L^{\prime}$ and $A^{\prime}$ on the covering $Q$. Then,

$$
\begin{aligned}
& L^{\prime} \cap \Omega=\left\{z_{3}+2 z_{1}^{2}=z_{1} z_{2}\right\}, \\
& A^{\prime} \cap \Omega=\left\{z_{2} z_{3}+z_{1}^{3}=0\right\},
\end{aligned}
$$

and

$$
L^{\prime} \cap A^{\prime} \cap \Omega=\left(s_{0} \cup \ell_{0}^{\prime}\right) \cap \Omega .
$$

Since $\left\{z_{2}=1\right\} \cap L^{\prime}=\left\{z_{3}+2 z_{1}^{2}=z_{1}\right\},\left\{z_{2}=1\right\} \cap A^{\prime}=\left\{z_{3}+z_{1}^{3}=0\right\}$, we find that $L^{\prime}$ and $A^{\prime}$ intersect transversally at the point $(0,0,1)$. This proves the lemma.

Q.E.D.

LEMMA 17. $L^{\prime}$ satisfies the criterion for contractibility.

Proof. Let $\ell^{\prime}$ be the proper transform of a generating line $\ell$ of $V$, and put $e=\left.\left[L^{\prime}\right]\right|_{L^{\prime}}$ (in $\left.X^{\prime}\right)$. We have only to show that $e \cdot \ell^{\prime}=-1$. In fact, since $L^{\prime}=\Psi^{*} V_{0}-Y^{\prime}, e=\left.\Psi^{*} V_{0}\right|_{L^{\prime}}-\left.Y^{\prime}\right|_{L^{\prime}}$. Therefore, $e \cdot \ell^{\prime}=s_{\infty} \cdot \ell^{\prime}-$ $\left(2 s_{0}+3 \ell^{\prime}\right) \ell^{\prime}=1-2=-1$, where $s_{\infty}$ is the section of $L^{\prime} \simeq \boldsymbol{F}_{2}$ with the self-intersection number 2 .

Q.E.D.

Let $\sigma: X^{\prime} \rightarrow X$ be the contraction of $L^{\prime}$. Then $X$ is a non-singular 3-fold and $\sigma\left(L^{\prime}\right)=L$ is a non-singular rational curve. We set $A=\sigma\left(A^{\prime}\right)$ and $E=\sigma\left(Y^{\prime}\right)$. Since $X^{\prime}-\Psi^{-1}\left(V_{0}\right) \simeq X^{\prime}-\left(L^{\prime} \cup Y^{\prime}\right) \simeq W-V_{0} \simeq C^{3}$ and $\sigma\left(L^{\prime}\right)=L \longrightarrow E$, we have $X-E=C^{3}$.

By the similar arguments to Proposition 1, we can see that the line bundle $[E]$ is positive on $X$ and $\operatorname{Pic} X \simeq Z \cdot c_{1}([E])$. On the other hand, $V$ is linearly equivalent to $V_{0}$, we have $\Psi^{*} V \sim \Psi^{*} V_{0}$, that is, $A^{\prime} \sim Y^{\prime}+L^{\prime}$ in $X^{\prime}$. Therefore $A \sim E$, namely, $A$ is linearly equivalent to $E$. By Lemmas $15,16, A$ is a singular Del Pezzo surface with an isolated singular point $\left\{a_{0}\right\}=\sigma\left(\ell_{0}^{\prime}\right)$. Since $K_{X^{\prime}}=-3 \Psi^{*} V_{0}+Y^{\prime}=-3 L^{\prime}-2 Y^{\prime}$, we have $K_{X}=-2 E=-2 A$. By Lemma $6, c_{1}\left(N_{Y \mid W}\right)=7$, hence $\left(Y^{\prime}\right)^{3}=-7$. Since $\left(L^{\prime}\right)^{3}=\left(\Psi^{*} V_{0}-Y^{\prime}\right)^{3}=V_{0}^{3}+3\left(\Psi^{*} V_{0} \cdot Y \cdot Y\right)-\left(Y^{\prime}\right)^{3}=2-9+7=0$, we have $c_{1}\left(N_{L \mid X}\right)=-\left(L^{\prime}\right)^{3}=0$. Thus $\left(c_{1}(X) \cdot L\right)=c_{1}\left(N_{L \mid X}\right)+2=2$, hence $(A \cdot L)=1$. Since $2=\left(A^{\prime}\right)^{3}=\left(\sigma^{*} A-L^{\prime}\right)^{3}=A^{3}-3(A \cdot L) A^{3}-3$, we have $A^{3}=5$. One can easily verify that $X$ is realized as a non-singular 3 -fold of degree 5 in $\boldsymbol{P}^{6}$, by the linear system $\left|\mathcal{O}_{X}(A)\right|$.

We shall finally show that $X-A=C^{3}$ In the affine part $\left\{w_{1}=1\right\} \simeq$ $C^{4}\left(w_{0}, w_{2}, w_{3}, w_{4}\right)$, we have 


$$
\begin{aligned}
& \hat{W} \simeq\left\{w_{2}^{2}=w_{0}+w_{3} w_{4}\right\} \\
& \hat{V}_{0} \simeq\left\{w_{4}=0\right\} \cap \dot{W} \\
& \dot{Y} \simeq\left\{w_{4}=0, w_{3}=w_{2}^{3}, w_{0}=w_{2}^{2}\right\} .
\end{aligned}
$$

We define a one to one regular mapping $\beta: C^{3}(x, y, z) \rightarrow C^{4}\left(w_{0} . w_{2}, w_{3}, w_{4}\right)$ by

$$
\left\{\begin{array}{l}
w_{0}=x^{2}-y z \\
w_{2}=x \\
w_{3}=y \\
w_{4}=z .
\end{array}\right.
$$

Then we have

$$
\begin{aligned}
& \hat{\beta}^{-1}(\stackrel{\circ}{W}) \simeq C^{3} \\
& \beta^{-1}\left(\stackrel{\circ}{0}_{0}\right) \simeq\{z=0\} \\
& \beta^{-1}(\stackrel{\circ}{Y}) \simeq\left\{y=x^{3}, z=0\right\} .
\end{aligned}
$$

By the coordinate transformation:

$$
\left\{\begin{array}{l}
u=x \\
v=y-x^{3} \\
w=z
\end{array}\right.
$$

we have

$$
\begin{aligned}
& \stackrel{\circ}{W} \simeq C^{3}(u, v, w) \\
& \stackrel{\circ}{V}_{0} \simeq\{w=0\} \\
& \stackrel{\circ}{Y} \simeq\{v=w=0\} .
\end{aligned}
$$

Let $\Psi^{\prime}: \dot{C}^{\prime} \rightarrow \stackrel{\circ}{W}$ be the blowing up of $\stackrel{\circ}{W}$ with center $\stackrel{\circ}{Y}$ above, and $L^{\prime \prime}$ be the proper transform of $\stackrel{\circ}{V}_{0}$. Then we have

$$
\stackrel{\circ}{W}^{\prime}-L^{\prime \prime} \cong X-A \text {. }
$$

By the definition of the blowing up, one can easily verify that $\dot{W}^{\prime}-L^{\prime \prime}$ $\cong C^{3}$, and thus $X-A \cong C^{3}$.

From the above arguments, we have also the following

TheOREN II. There exists an projective algebraic compactification $(X, E)$ of $C^{3}$ such that $E$ is irreducible but not normal.

Proof. Under the same notations as above, we put $E=\sigma\left(Y^{\prime}\right)$. Then we have seen that $X-E \cong C^{3}$ and $E$ is irreducible (see Lemma 9). Since 
$E$ is a ruled variety swept out by lines which intersect $L, E$ contains a plenty of lines. By Proposition 15, $E$ is not normal. Q.E.D.

(4.4) Non-existence of $(X, A)$ with $m=6$.

By Iskovski [11] (N.B.: Iskovski's result on $\left(-K_{X} / 2\right)^{3}=6$ misses one case, see T. Fujita, On the structure of polarized manifolds with total deficiency one, I, J. Math. Soc. Japan 32 (1980) 707-725; II, 33 (1981) 415434.) it is shown that a Fano 3 -fold $X$ with index 2 and Pic $X \cong Z$ satisfies $\left(-K_{X} / 2\right)^{3} \leqq 5$. Therefore $m \neq 6$.

(4.5) Conclusion. Let $(X, A)$ be a non-singular Kahler compactification of $C^{3}$ such that $A$ has at most isolated singular points. If $r=2$, then $X$ is a non-singular 3 -fold of degree 5 in $\boldsymbol{P}^{6}$ and $A$ is a tangent hyperplane section which is normal. Further, such a $(X, A)$ is determined uniquely up to isomorphism. The detailed construction of this $(X, A)$ is given in (4.3) of this section.

\section{REFERENCES}

[1] E. Bombieri and D. Husemoller, Classifications and embeddings of surfaces, Algebraic Geometry, Arcata 1974, Amer. Math. Soc. Proc. Symp. Math., Providence, 29 (1975), 329-420.

[2] L. Brenton, Some algebraicity criteria for singular surfaces, Invent. Math., 4 (1977), 129-144.

[3] - On singular complex surfaces with negative canonical bundle, with applications to singular compactifications of $C^{2}$ and to 3-dimensional rational singularities, Math. Ann., 248 (1980), 117-124.

[ 4 ] L. Brenton, D. Drucker and G. C. E. Prince, Graph theoretic techniques in algebraic geometry II: Construction of singular complex surfaces of rational cohomology type of $\boldsymbol{C P}^{2}$ Comment. Math. Helv., 56 (1981), 39-58.

[5] L. Brenton and J. Morrow, Compactifications of $C^{n}$ Trans. Amer. Math. Soc., 246 (1979), 139-158.

[6] M. Demazure, Surfaces de Del Pezzo, Lecture Note. in Math., 777 (1980), 23-69, Springer-Verlag Berlin Heidelberg New York.

[7] P. Griffiths and J. Harris, Principles of Algebraic Geometry, Pure and Applied Math., John Wiley and Sons, New York, 1978.

[8] R. Hartshorne, Algebraic Geometry, Graduate Texts in math. 52, Springer-Verlag, New York Heidelberg Berlin, 1977.

[9] F. Hidaka and K. Watanabe, Normal Gorenstein surfaces with ample anticanonical divisor, Tokyo J. Math., 4 (1981), 319-330.

[10] F. Hirzebruch, Topological Methods in Algebraic Geometry, Grundlehren der math. Wissenschaften 131, Springer-Verlag Berlin Heidelberg New York, 1966.

[11] V. A. Iskovski, Fano 3-fold I, Math. U.S.S.R. Izvestija, 11 (1977), 485-527.

[11 $\mathrm{a}$ - Anticanonical models of three-dimensional algebraic varieties, J. Soviet Math., 13-14 (1980), 745-814.

[12] K. Kodaira, On compact analytic surfaces II, Ann. Math., 77 (1963), 536-626. 
[13] S. Kobayashi and T. Ochiai, Characterizations of complex projective spaces and hyperquadrics, J. Math. Kyoto Univ., 13 (1973), 31-47.

[14] H. Laufer, Normal Two-Dimensional Singularities, Ann. Math., Studies 71, 1971.

[15] J. Morrow, Minimal normal compactifications of $C^{2}$, Proc. conf. of Complex Analysis, Rice Univ. Studies, 59 (1973), 97-112.

[16] D. Mumford, Varieties defined by quadratic equations, Questions on algebraic varieties, Edizioni Cremonese, Rome, 1970, 63-82.

[17] R. Remmert and T. Van de Ven, Zwei Satze über die komplex-projektive ebene, Niew Arch. Wisk., 8 (3) (1960), 147-157.

[18] B. Saint-Donat, Projective model of K3 surfaces, Amer. J. Math., 96 (1974), 602639.

[19] K. Saito, Einfach-elliptische Singularitäten, Invent. Math., 23 (1974), 289-325.

[20] P. Wagreich, Elliptic singularities of surfaces, Amer. J. Math., 92 (1970), 419-454.

Kumamoto Radio Technical College

2659-2, Suya, Nishigoshi-machi,

Kikuchi-gun, Kumamoto 861-11

Japan 